\title{
Identification of the feline foamy virus Bet domain essential for APOBEC3 counteraction
}

\author{
Dragana Slavkovic Lukic ${ }^{1}$, Agnes Hotz-Wagenblatt ${ }^{2}$, Janet Lei ${ }^{1}$, Ann-Mareen Räthe', Michael Mühle \\ Joachim Denner ${ }^{3}$, Carsten Münk ${ }^{4}$ and Martin Löchelt ${ }^{1 *}$
}

\begin{abstract}
Background: APOBEC3 (A3) proteins restrict viral replication by cytidine deamination of viral DNA genomes and impairing reverse transcription and integration. To escape this restriction, lentiviruses have evolved the viral infectivity factor (Vif), which binds A3 proteins and targets them for proteolytic degradation. In contrast, foamy viruses (FVs) encode Bet proteins that allow replication in the presence of A3, apparently by A3 binding and/or sequestration, thus preventing A3 packaging into virions and subsequent restriction. Due to a long-lasting FV-host coevolution, Bet proteins mainly counteract restriction by A3s from their cognate or highly related host species.

Results: Through bioinformatics, we identified conserved motifs in Bet, all localized in the bel2 exon. In line with the localization of these conserved motifs within bel2, this part of feline FV (FFV) Bet has been shown to be essential for feline A3 ( $\mathrm{feA}$ ) inactivation and feA3 protein binding. To study the function of the Bet motifs in detail, we analyzed the ability of targeted deletion, substitution, and chimeric FFV-PFV (prototype FV) Bet mutants to physically bind and/or inactivate feA3. Binding of Bet to feA3Z2b is sensitive to mutations in the first three conserved motifs and $\mathrm{N}$ - and C-terminal deletions and substitutions across almost the complete bel2 coding sequence. In contrast, the Bel1 (also designated Tas) domain of Bet is dispensable for basal feA3Z2b inactivation and binding but mainly increases the steady state level of Bet. Studies with PFV Bel1 and full-length FFV Bel2 chimeras confirmed the importance of Bel2 for A3 inactivation indicating that Bel1 is dispensable for basal feA3Z2b inactivation and binding but increases Bet stability. Moreover, the bel1/tas exon may be required for expression of a fully functional Bet protein from a spliced transcript.

Conclusions: We show that the Bel2 domain of FV Bet is essential for the inactivation of APOBEC3 cytidine deaminase restriction factors. The Bel1/Tas domain increases protein stability and can be exchanged by related sequence. Since feA3 binding and inactivation by Bet are highly correlated, the data support the view that FV Bet prevents A3-mediated restriction of viral replication by creating strong complexes with these proteins.
\end{abstract}

Keywords: APOBEC3, Retrovirus, Foamy virus, Antiviral restriction factors, Bet protein, Host-virus interaction, Virus defence protein

\section{Background}

Cellular restriction factors are key players of intrinsic immunity, which acts against viruses immediately after infection [1]. Restriction factors are constitutively expressed in cells but their expression can be increased by interferons produced upon viral infection [2-7]. So far, four anti-retroviral restriction factors have been functionally

\footnotetext{
* Correspondence: m.loechelt@dkfz.de

'Research Program Infection and Cancer, Department Genome Modifications and Carcinogenesis, German Cancer Research Center (DKFZ), Heidelberg, Germany

Full list of author information is available at the end of the article
}

characterized in detail: APOBEC3, Trim $5 \alpha$, tetherin and SAMHD1 [8-12]. In addition, several other restriction factors have been detected by genome-wide screens but require additional experimental characterization [13,14]. Restriction factors interfere with defined steps in the viral life cycle, leading to attenuation or complete suppression of virus replication. By coevolution with host-encoded restriction factors, current viruses have achieved mechanisms to circumvent this inhibitory activity. Some viruses, for instance, have acquired special proteins that directly counteract restriction factors $[1,2,8,12,15]$. Due to the interaction of viral proteins with restriction factors, both

\section{Biomed Central}


viral and host-encoded proteins are under constant positive selection to evade or strengthen, respectively, this functional interaction [16]. Host-virus coevolution directly impacts the species-specificity of a given pathogen and is considered as one of the main factors preventing interspecies virus transmission [2].

APOBEC3 (A3) cytidine deaminases are potent antiviral restriction factors. These host proteins deaminate cytidine residues in single-stranded (ss) DNA intermediates generated during retroviral reverse transcription, creating hypermutated genomes with uridine residues [17]. Although considered to deaminate only ssDNA, A3 proteins are also active against double-stranded DNA viruses such as papillomaviruses, probably due to a transient exposure of ssDNA during replication and/or transcription [18]. In addition, it has been shown that A3s deaminate genomes of hepadnaviruses, endogenous retroviruses, retroid elements and possibly even cellular genes [8,19-22]. To restrict retroviral replication, A3 restriction factors must be incorporated into viral particles to edit the forming proviral DNA in the newly infected cell [23]. In contrast, A3s deaminate foamy virus (FV) genomes not only in newly infected cells, but also in virus-producing cells [15] as reverse transcription of FVs may already occur in virus-producing cells [24]. In addition, it has been shown that some human A3s have deaminase-independent activities such as blocking reverse transcription and integration [25-31]. In fact, A3 proteins with mutated catalytic sites have been shown to be active against HIV-1 [25,31,32].

The dynamic coevolution of host defences and virus counter-defence has resulted in a significant expansion of the A3 locus in higher mammals by distinct gene duplication events. In humans, this has led to seven distinct genes/proteins (A3A, B, C, DE, F, G, H), while only four single-domain A3 genes are present in cats [33-37]. Additional complexity of the A3 repertoire in cats is achieved by alternative and complex splicing events of these genes leading to one two-domain feline A3 (feA3) proteins; for details, see refs. [33,37,38].

Two classes of retroviral counter-defence proteins against A3s have been described so far: lentivirus Vif and FV Bet. Data on how other retroviruses replicate in the presence of A3 restriction factors is scarce, but indicate that these viruses have developed other means to avoid A3 packaging [27,39].

The mechanism of A3 inactivation by lentiviral (HIV) Vif is well characterized. Vif acts as an adaptor protein that binds to both $\mathrm{A} 3$ and ubiquitin ligase complexes consisting of cullin-5, RING-box 1 and elongins B and C [40]. Thus, Vif induces the ubiquitination and subsequent degradation of A3 by the proteasome [40]. It has been shown that $\mathrm{N}$ terminal regions of $\mathrm{Vif}$ bind to huA3G and huA3F while a SOCS box motif mediates ubiquitin ligase complex binding [40,41]. Binding of Vif to A3G is species-specific and substitution of only a single amino acid in human A3G results in a mutant that is no longer targeted by HIV Vif [42].

The FV bet genes are expressed by all known exogenous FVs but are also present in the sloth endogenous FV (SloEFV) genome, which is at least 100 million years old [43]. Feline and prototype/primate/human FV (PFV) Bet have been recently shown to counteract defined A3 proteins of feline and human/non-human primate (NHP) origin, resp., and protect FV replication in A3-positive cells $[15,44,45]$. There is no sequence homology between Bet and Vif and Bet does not contain the SOCS box motif required for E3 ubiquitin ligase complex interactions [46]. In contrast to lentiviral Vif, Bet does not induce A3 degradation $[15,44,47]$. Bet is thought to directly bind and possibly sequester A3 proteins, preventing their incorporation into viral particles $[15,44]$. In line with this, Bet is expressed at high levels in infected cells and animals [48] which may be a prerequisite for such a stoichiometric reaction.

Bet is an accessory protein of FVs, viruses that display a complex genetic organization. Together with the essential Bel1/Tas transactivator of both FV promoters, Bet is mainly expressed from the internal promoter located in the 3 'end of env [49]. Bet is the product of a splicing event that fuses the $5^{\prime}$ domain of bel1 to the complete bel2 open reading frame (ORF). All known FVs encode bet and, as shown by genome localization, corresponding bet genes are also present in endogenous FVs [43,50]. A protein consisting of bel2 only is not expressed in vitro; whether it is expressed in vivo is still unknown.

Although it has been shown that FFV Bet binds to all known feA3 proteins, the amino acid residues involved in binding have yet to be identified. It has been shown, however, that FFV Bet-MCS, with a mutation in the Bel2 domain of Bet, is incapable of counteracting feA3 and cannot replicate in A3-positive CrFK cells [15,47,51]. In this FFV proviral genome, a multiple cloning site (MCS) had been introduced inside the bel2 ORF, leading to the alteration of $\mathrm{E}^{117} \mathrm{~L}^{118} \mathrm{~L}^{119}$ residues to ASVRRGP [51]. Despite complete sequence conservation in the rest of Bet, Bet-MCS does not bind or inactivate feA3s, indicating that the mutated region is important for A3 counteraction $[15,47]$. Not surprisingly, the replication of FFV-BBtr, which contains only a truncated bet gene, is likewise strongly impaired in the presence of A3s [15,47,51].

FVs are retroviruses that differ from other viruses of this group in many aspects such as protein processing, morphogenesis, gene expression, and replication [49,52,53]. FVs have not yet been associated with any disease and are considered apathogenic [54]. This feature makes FVs potential vectors for gene delivery and vaccination [53]. The known human FV isolates are results of zoonotic transmissions of diverse simian FVs to humans [55]. With respect to virus-host coevolution, FV show a very strong coevolution with their host and related species [56,57]. 
At current, FV research focuses mostly towards all aspects of vector development, host-virus coevolution and the potential of interspecies transmission to other hosts, including humans [54].

In this study, we analyzed the functional interaction between FFV Bet and feA3 restriction factors as a model for the situation in humans and NHPs, since the feA3 repertoire is less complex than that of these species. Using bioinformatics and reverse genetics, we identified conserved motifs in Bet and tested their importance. We identified the bel2 sequence of Bet as the essential determinant for A3 inactivation. Moreover, this study shows that nearly the entire FFV Bel2 domain is required for feA3 binding and inactivation, supporting the view that Bet inactivates feA 3 by creating strong complexes with this restriction factor $[44,45,47]$.

\section{Results}

\section{Bet contains six conserved motifs}

All known FV Bet proteins consist of the N-terminus of Bel1 and the complete Bel2 sequence. However, sequence homology between Bet proteins of different FVs is very low
[49]. To identify any possible conserved motifs amongst different Bet proteins, we performed bioinformatics using the MEME program, which searches for repeated amino acid patterns in given sequences [58]. Six conserved motifs, all localized within Bel2, were identified in the Bet proteins of bovine (BFV), equine (EFV), simian (SFV) FVs, FFV, PFV and SloEFV (Figure 1). MEME detected four motifs with standard settings (shown in black, motifs 1, 2, 3, 5); two additional motifs ( 4 and 6 , marked in gray letters) were identified by searching with only the bel 2 sequences and using slightly modified parameters. The first five motifs are also conserved in the predicted Bet sequence of SloEFV, although the third and fifth motifs from SloEFV appear to be shorter than the corresponding motifs in other Bet proteins (Figure 1). In EFV Bet, the positions of the second and the third motifs are swapped with respect to the corresponding motifs in the other FV Bet proteins.

\section{The Bel2 part of FFV Bet is sufficient for feA3Z2b binding and inactivation}

It was previously shown that FFV Bet binds and inactivates diverse feA3 proteins [15,47]. To determine the

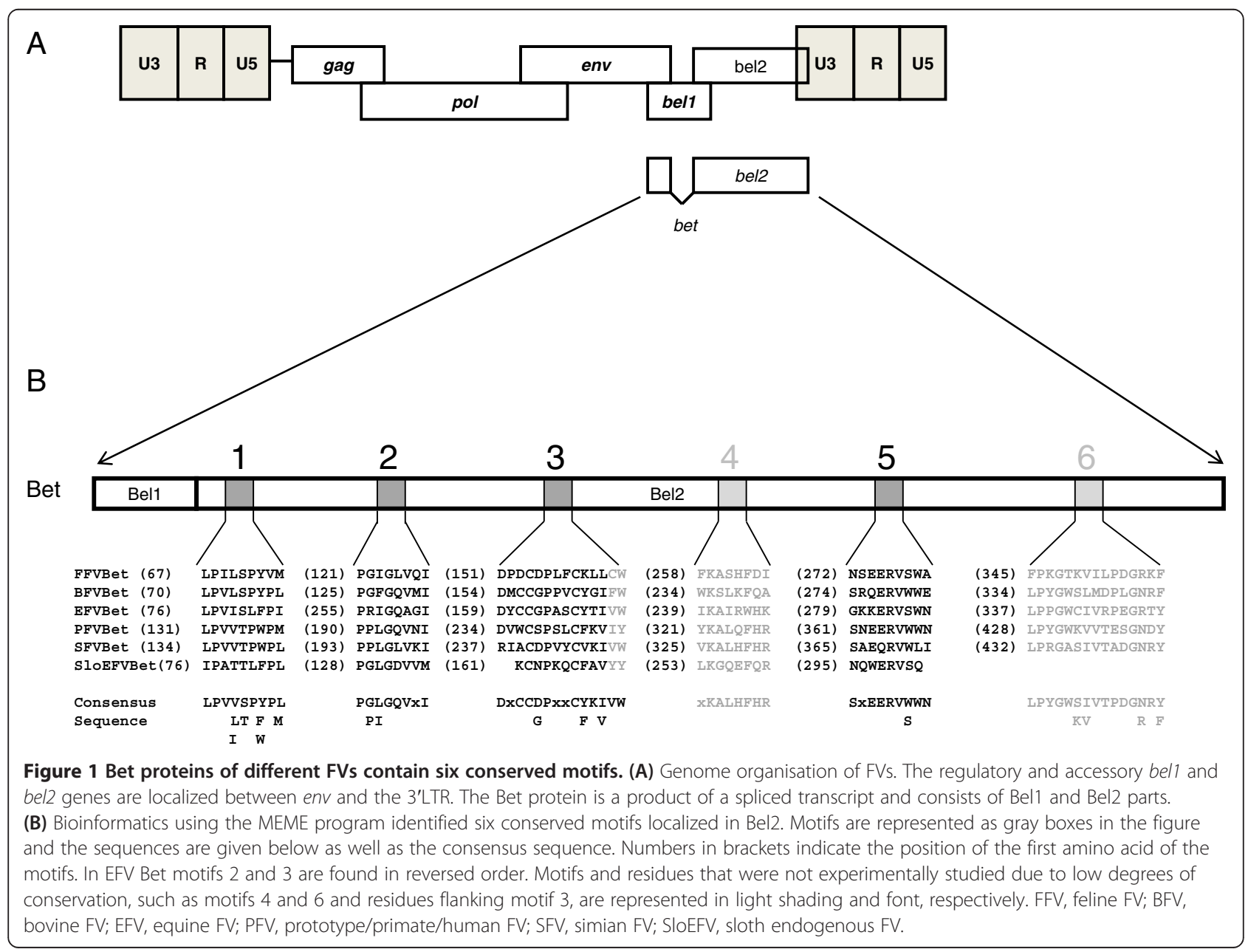


minimal FFV Bet sequence required for these two functions, N-terminal Bet deletion mutants were constructed (Figure 2A). Eukaryotic expression clone Bel2ORF encompasses the whole bel2 ORF, while Bel2ATG starts with the first start codon inside bel2 which is not conserved among known FVs. Bel2ORF contains all six conserved motifs, while Bel2ATG and mutants Bet $\Delta$ N82 and Bet $\Delta$ N92 lack the first motif.
We tested the ability of the mutants to bind to and/or inactivate feA3Z2b, the feA3 with the highest restriction potential against Bet-deficient FFV (FFV-BBtr) [33,47,51]. HEK293T cells were cotransfected with pCF-BBtr, the empty vector pcDNA, or pcfeA3Z2b-HA, and increasing amounts of plasmids encoding either wt or mutant Bet (Figure 2B). Two days post transfection (d.p.t.), viral infectivity was determined by titration using FeFab cells.

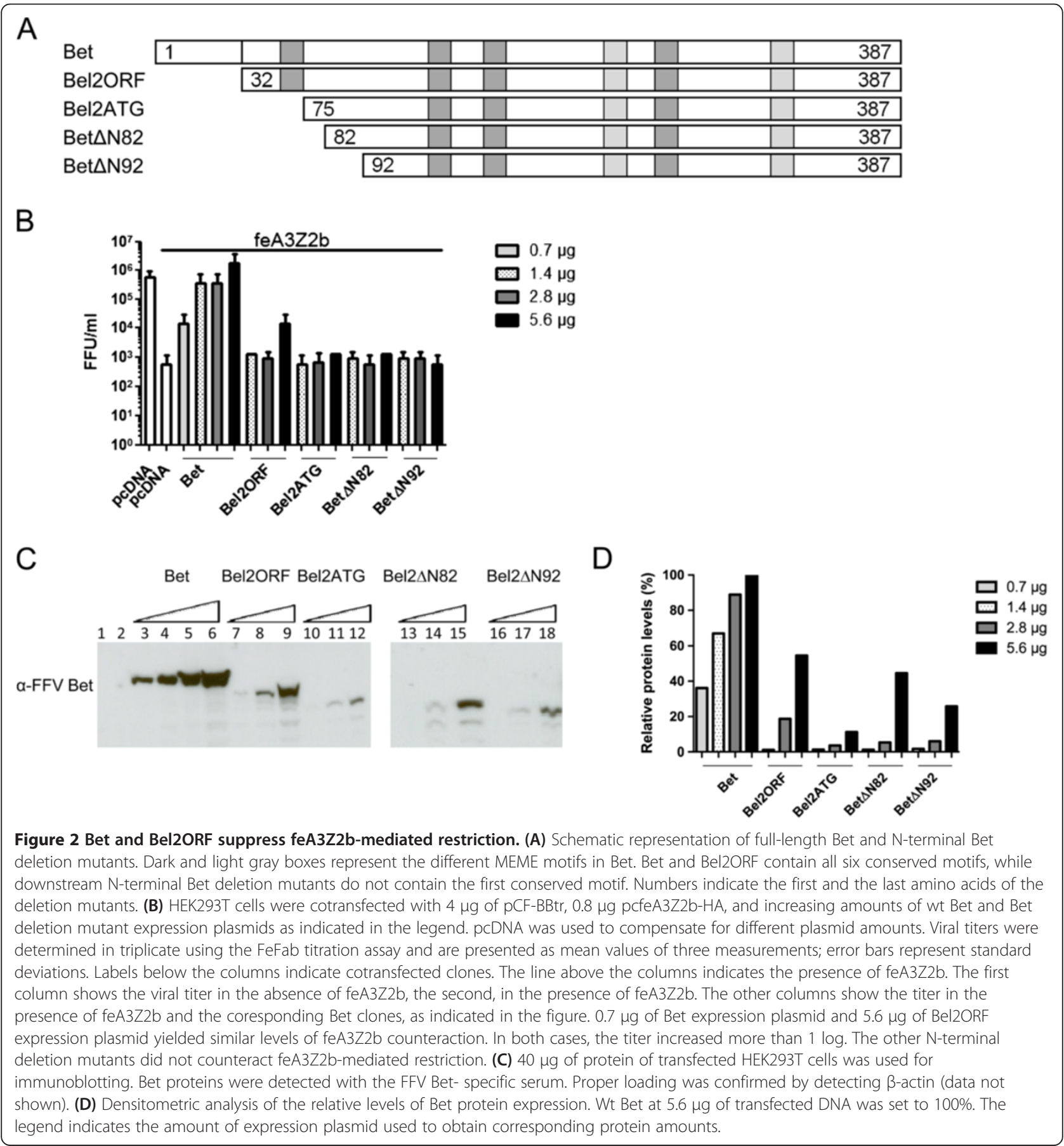


Figure $2 \mathrm{~B}$ shows that feA3Z2b resulted in a 3-log decrease of viral titer. Wt Bet efficiently counteracted this restriction in a dose-dependent manner. The highest concentration of Bel2ORF partially increased viral titer in the presence of feA3Z2b, while shorter deletion mutants did not have any effect on the viral titer. The presence of Bet in cell lysates was confirmed by immunoblotting with an FFV Bet-specific serum (Figure 2C). Levels of Bet deletion mutants were lower relative to the level of wt Bet, indicating instability of the deletion mutants (Figure $2 \mathrm{C}$ and $\mathrm{D}$ ). As inactivation of feA3Z2b by Bet is concentrationdependent, the lower potential of Bel2ORF to counteract feA3Z2b may be due to the lower steady state of deletion mutants. As shown in Figure 2 panels B and D, at 0.7 and $5.6 \mu \mathrm{g}$ transfected plasmid encoding Bet or Bel2ORF, respectively, slightly increased amounts of Bel2ORF protein resulted in comparable restoration of infectivity of the betdeficient FFV genome in the presence feA3Z2b. Both wt and truncated Bet were detected with similar efficacy by the Bet antiserum and the V5 tag antibody, shown by comparative immunoblotting (Additional file 1).

The ability of the truncated Bet proteins to bind feA3Z2b was tested in pulldown assays with bacterially expressed glutathione-S-transferase (GST) or GST-tagged feA3Z2b and lysates from HEK293T cells expressing wt or mutant Bet, as described previously [47]. Levels of N-terminal Bet deletion mutants were increased by supplementing the cell culture medium with $8 \mathrm{mM}$ sodium butyrate. Bound proteins were precipitated using glutathione-coupled beads and detected by immunoblotting. In addition to wt Bet, known to bind to feA3Z2b [15,47], Bel2ORF was efficiently precipitated with feA3Z2b (Figure 3). Although low intensity bands are present in the case of Bel2ATG and Bet $\Delta \mathrm{N} 82$, they were not reproducible and therefore considered to be unspecific. To confirm proper setup of the assays, the presence of similar amounts of GST and GSTfeA3Z2b was confirmed with an SV40 tag-specific antibody (Figure 3 lower panel).

\section{C-terminal Bet deletion mutants do not counteract feA3Z2b-mediated restriction}

To determine the minimal Bet sequence required for A3 inactivation, $\mathrm{C}$-terminal deletion mutants were constructed (Additional file 2A). In transfected cells, the engineered proteins were present at only very low levels and thus, functional studies were conducted in the presence and absence of the proteasome inhibitor ALLN (Additional file 2B). HEK293T cells were transfected with pCF-BBtr, feA3Z2b, and wt or mutant Bet constructs. Cultures were treated for approximately $24 \mathrm{~h}$ with $25 \mu \mathrm{M}$ of the proteasome inhibitor ALLN and tested for their ability to counteract feA3Z2b restriction. Both V5-tagged and untagged full-length Bet but none of the C-terminal Bet deletion mutants were able to inactivate feA3Z2b in the presence of ALLN or DMSO (solvent control) (Additional file 2B). The levels of wt Bet and wt Bet-V5 proteins did not change in

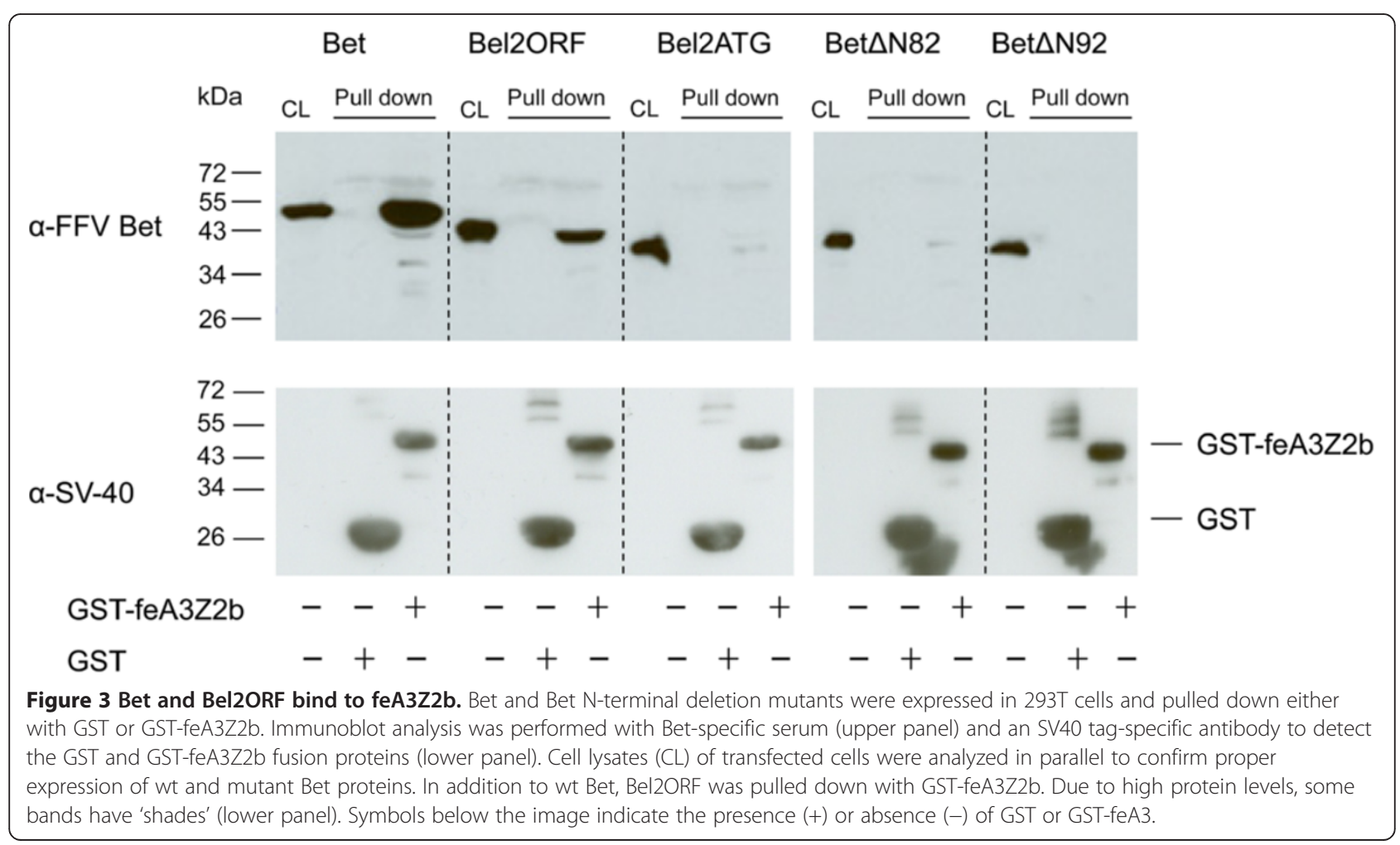


the presence of ALLN, while the level of C-terminal deletion mutants increased due to proteasome inhibition (Additional file 2C).

The feA3Z2b binding ability of the C-terminal deletion mutants was also tested in pulldown assays. None of the C-terminal deletions bound to feA3Z2b, despite increased expression levels in transfected cells due to sodium butyrate addition (Additional file 2D).

\section{PFV Bet does not counteract or bind to feline A3Z2b}

To circumvent the problems of the low stability of truncated FFV Bet proteins, we constructed chimeric FFVPFV proteins. Bet proteins from different FVs are known to counteract A3s from cognate species but not from distantly related species. For instance, it has previously been shown that FFV Bet does not bind to human A3s [15] and that PFV Bet does not inactivate murine A3 $[44,45]$. We therefore first determined whether PFV Bet binds or inactivates feA3Z2b. HEK293T cells were cotransfected with pCF-BBtr, pcfeA3Z2b-HA or pcDNA, and plasmids expressing FFV or PFV Bet. As shown in Figure 4A, viral titers determined two d.p.i. decreased in the presence of feA3Z2b and were completely rescued by FFV Bet while, as anticipated, PFV Bet did not show anti-feA3 activity.

Since PFV Bet bound to glutathione beads incubated with GST (data not shown), the pulldown assay described above was not suitable for studying PFV Bet-feA3Z2b interactions, we thus used coimmunoprecipitation (co-IP) assays instead. HEK293T cells were cotransfected with pcfeA3Z2b-HA and plasmids expressing PFV or FFV Bet.
Two d.p.t., co-IP was performed with monoclonal antiHA IgG. Precipitated proteins were detected by immunoblotting. Unlike FFV Bet, PFV Bet did not coimmunoprecipitate with feA3Z2b-HA (Figure 4B and C). Immunoblotting with anti-HA IgG showed the presence of feA3Z2b-HA and an unspecific band of lower molecular mass (Figure $4 \mathrm{~B}$ and $\mathrm{C}$, lower panel). Incubation of cell lysates with beads only (without antibody, mock co-IP) did not result in unspecific feA3Z2b-HA precipitation.

\section{Chimeric FFV-PFV Bet proteins containing almost the entire FFV Bel2ORF bind and inactivate feA3Z2b}

To stabilize FFV Bet deletion mutants, eleven chimeric FFV-PFV Bet proteins, equivalent to the Bet deletion mutants described above, were constructed. In these chimeric proteins, deleted parts of FFV Bet were substituted by the equivalent parts of PFV Bet (Figure 5).

Both PFV and FFV Bet consist of Bel1 and Bel2 regions (Figure 5A), though the sizes of Bel1 regions of FFV and PFV Bet differ greatly, between 31 and 88 amino acids. In order to mimic this situation, three chimeric proteins containing the full FFV Bel2ORF and different lengths of PFV Bel1 were cloned as described in Methods. FFV-PFV $\mathrm{CH} 1$ contains the first $\mathrm{N}$-terminal 39 amino acids of PFV Bel1 fused to the FFV Bel2ORF and is still 8 amino acids larger than the FFV Bel1 part but maintains a predicted extended secondary structure in the PFV sequence (data not shown). FFV-PFV CH2 contains PFV Bel1 and four additional residues from PFV Bel2 to avoid deletion of a predicted alpha helix in PFV Bet (data not shown).

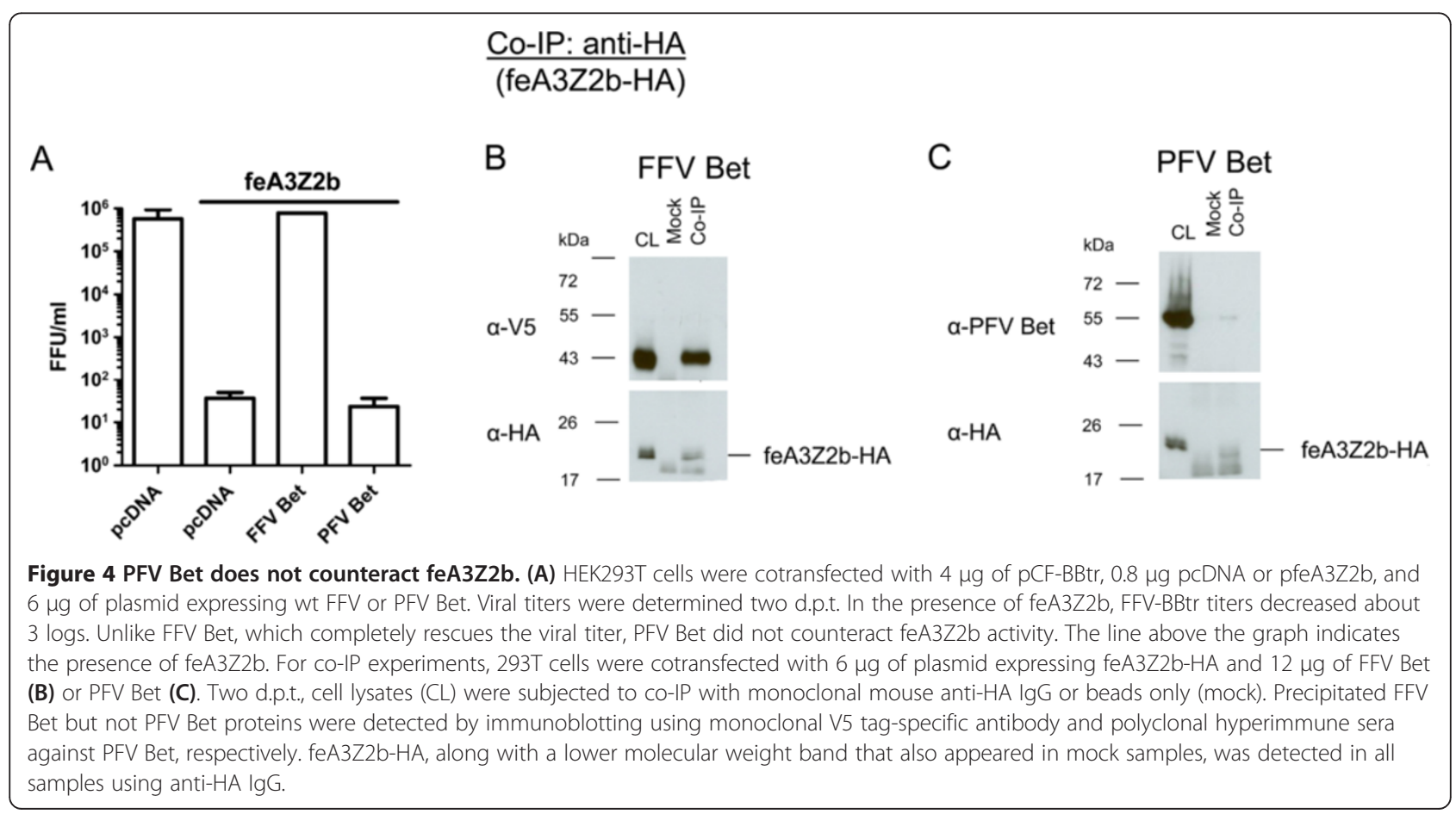




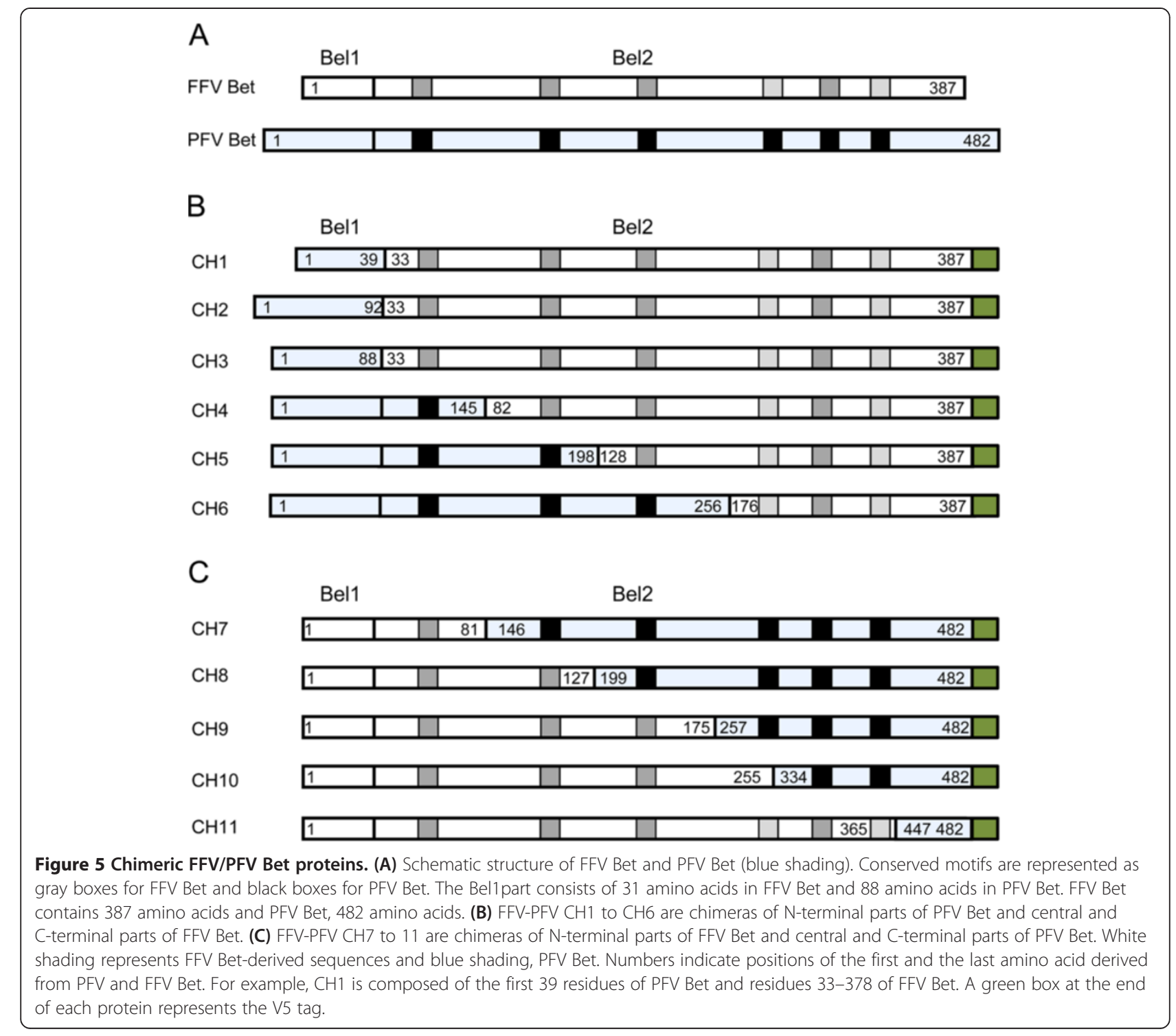

FFV-PFV CH3 contains the entire PFV Bel1 part fused directly to FFV Bel2ORF. FFV-PFV CH4 to 6 contain shorter C-terminal FFV Bet and longer N-terminal PFV Bet segments, while chimeric proteins $\mathrm{CH} 7$ to 11 are equivalent to FFV Bet C-terminal deletion mutants (Figure 5C). All chimeric proteins were V5-tagged to facilitate proper protein detection (Figure 5B and C).

To determine whether chimeric proteins $\mathrm{CH} 1$ to 6 inactivate feA3Z2b, HEK293T cells were cotransfected with pCF-BBtr, pcfeA3Z2b-HA, and plasmids encoding either wt PFV Bet, wt FFV Bet, or one of the chimeric FFV-PFV Bet $\mathrm{CH} 1$ to $\mathrm{CH} 6$ proteins. Viral titers determined 2 d.p.t. are shown in Figure 6A. As shown before, PFV Bet did not counteract feA3Z2b activity, while chimeric proteins $\mathrm{CH} 1$ to 3, containing the full-length FFV Bel2ORF and different PFV Bel1 sequences, fully restored FFV titers to levels similar to wt Bet (Figure 6A). Bet chimeras 4 to 6 , with $\mathrm{N}$-terminal FFV Bel2 sequences replaced by those from PFV, were non-functional and did not suppress feA3Z2b restriction.

FFV-PFV chimeric proteins $\mathrm{CH} 7$ to 11 , carrying Cterminal PFV sequences of decreasing size were characterized as described above. Only FFV-PFV Bet CH11, carrying a short and obviously non-conserved C-terminal PFV Bet fragment, was functionally active against feA3Z2b.

The presence of proteins in cell lysates was confirmed by immunoblotting with the V 5 antibody. Figure $6 \mathrm{~B}$ and $6 \mathrm{D}$ show that the levels of chimeric proteins were comparable or slightly lower than those of wt FFV Bet. The PFV Bet antiserum efficiently detected full-length Bet but 


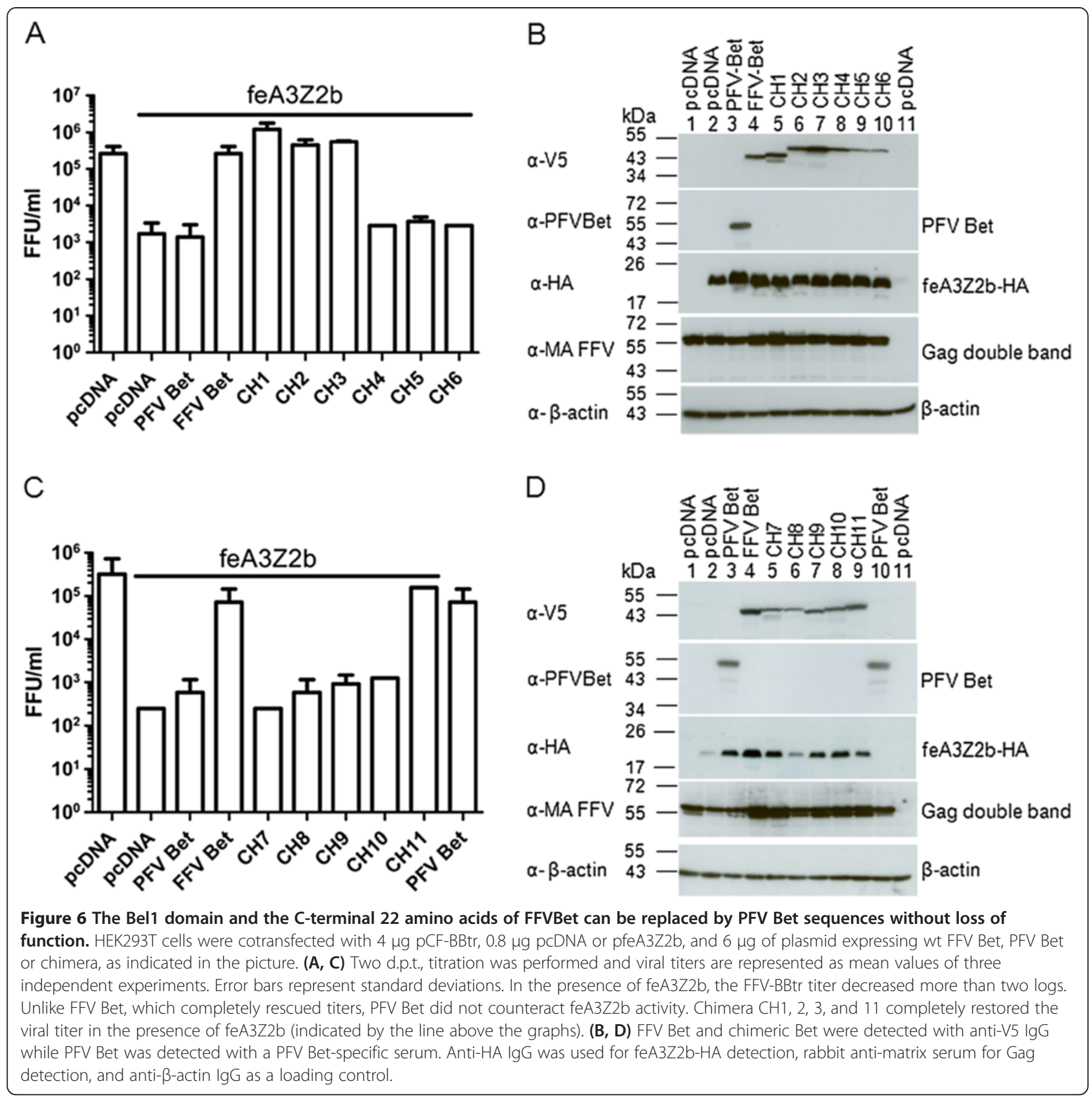

not chimera $\mathrm{CH} 1$ to $\mathrm{CH} 5$ and $\mathrm{CH} 10$ and $\mathrm{CH} 11$. In contrast, $\mathrm{CH} 6$ to $\mathrm{CH} 9$ were detectable only upon extended exposure (not shown).

Binding of chimeric FFV-PFV Bet proteins to feA3Z2b was studied by co-IPs as described above. As shown in Figure 7A and B, only FFV-PFV chimeric proteins $\mathrm{CH} 1$, 2, 3 and 11 coimmunoprecipitated with feA3Z2b-HA. Importantly, unspecific binding to the beads (mock coIP) was not observed. Proper set up of the assay was confirmed by detection of feA3Z2b-HA (Figure 7, labelled $\alpha-\mathrm{HA}$ in parts $\mathrm{A}$ and $\mathrm{B})$.
Identification of critical Bet residues by alanine scanning mutagenesis of conserved motifs

To identify functionally important amino acids in the conserved Bet motifs, site-directed alanine scanning mutagenesis was performed. Amino acids in the conserved motifs or in flanking sequences were substituted by alanine as described in Methods and indicated in Figure 8, resulting in $20 \mathrm{FFV}$ Bet mutants carrying one to three amino acid substitutions each. Although these Bet mutants are not classical deletion mutants, $\Delta$ symbols were used to facilitate labelling of these substitutions. 


\section{Co-IP: anti-HA $(\mathrm{feA3Z2b-HA)}$}

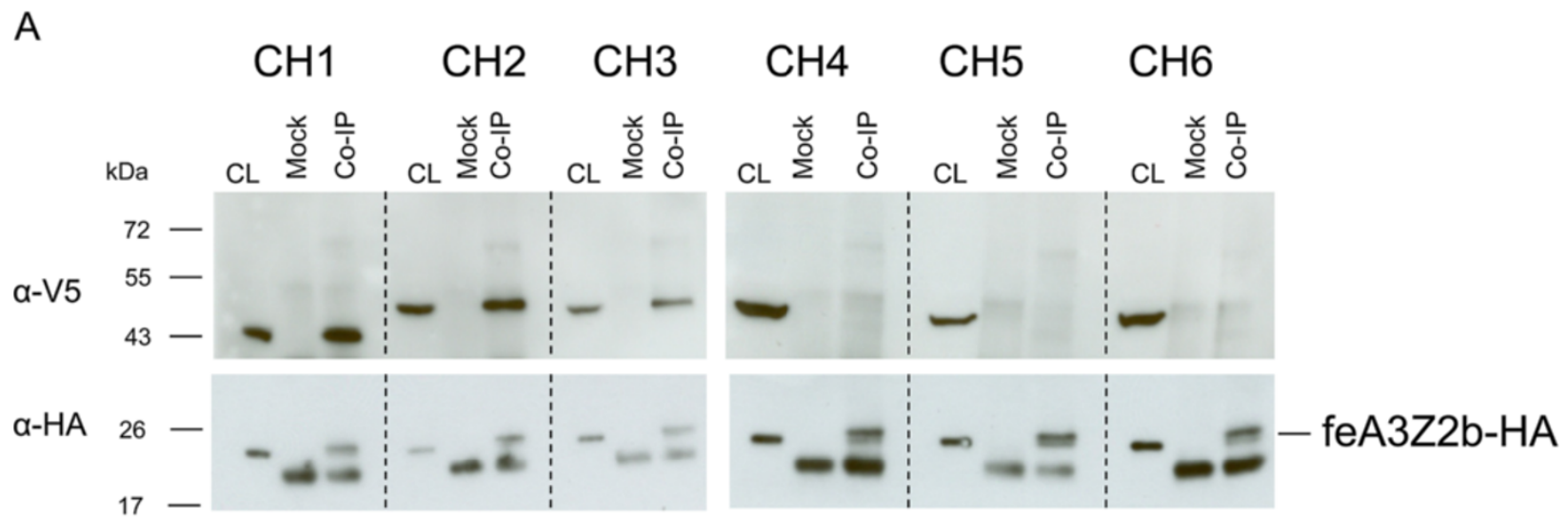

B

$\begin{array}{llllll}\mathrm{CH} 7 & \mathrm{CH} 8 & \mathrm{CH} 9 & \mathrm{CH} 10 & \mathrm{CH} 11 & \text { pcDNA }\end{array}$
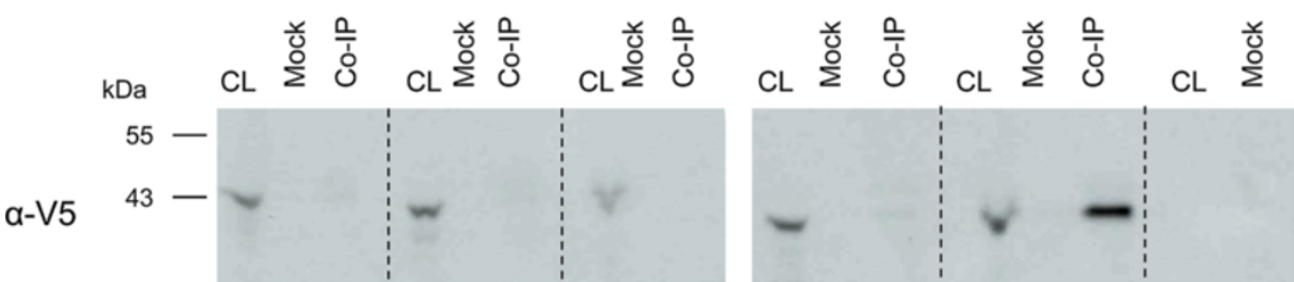

a-HA
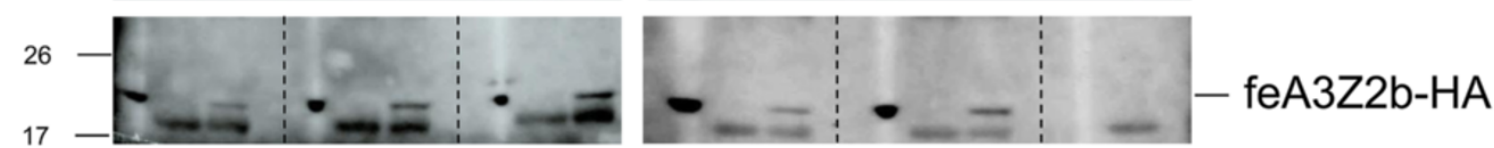

Figure 7 FFV-PFV CH1, CH2, CH3 and CH1 1 bind to feA3Z2b-HA. 293T cells were cotransfected with $6 \mu$ g of plasmid expressing feA3Z2b-HA and $12 \mu \mathrm{g}$ of one of the chimeric protein expression plasmids (A, N-terminal chimera $\mathrm{CH} 1$ to CH6; $\mathbf{B}$, C-terminal chimera $\mathrm{CH}$ to $\mathrm{CH} 11)$ or pcDNA, as indicated in the picture. Two d.p.t., cell lysates $(\mathrm{CL})$ were subjected to co-immunoprecipitation (co-IP) with monoclonal anti-HA IgG or beads only (mock). Precipitated proteins were detected by immunoblotting. feA3Z2b-HA was detected with an anti-HA monoclonal antibody in each sample, as expected. A lower molecular weight band that was also detected is considered unspecific, as it also appeared in mock samples. Hatched lines mark empty lanes used to separate individual experiments. Chimeric proteins were detected with anti-V5 IgG. In addition to FFV Bet, $\mathrm{CH} 1,2,3$, and 11 were coimmunoprecipitated by feA3Z2b.

The second conserved FFV Bet motif is localized directly behind the mutated part of FFV Bet-MCS, in which an MCS had been inserted inside bel2 [51]. The MCS introduction resulted in the substitution of three and addition of four amino acids. Although the rest of Bet remained unchanged, Bet-MCS did not have anti-feA3 activity and did not bind to feA3 $[15,47,51]$. We therefore proceeded to more carefully characterize the second conserved motif together with these flanking sequences.

Functional feA3 inactivation and binding studies by GST-feA3Z2b pulldown were performed to determine whether mutant Bet proteins inactivate and/or bind feA3Z2b, as described above. The results are summarized in Figure 8 and provided in Additional files 3 and 4. As indicated in Figure 8, most mutations in or close to the first, second, or third motifs were detrimental to both Bet functions, while all 3 amino acid replacement mutants of the fifth motif displayed a wt phenotype with respect to feA3 inactivation and binding. Moreover, FFV Bet function was not impaired by single amino acid changes, while double amino acid mutations in motif 2 resulted in loss of function (Figure 8 and Additional file 3). In fact, Bet $\Delta V$ and Bet $\Delta \mathrm{P}$ were both functionally active but the double mutant Bet $\Delta \mathrm{VP}$, in which both amino acids are replaced by alanine, was completely incapable of binding and counteracting feA3Z2b. As indicated in Figure 8 and shown in the Additional file 4, all mutants capable of functionally inactivating feA3Z2b also bound to this restriction factor in pulldown assays. In addition, Bet $\Delta \mathrm{LTM}$ where mutations are outside of motif 2 and Bet $\Delta \mathrm{I}$ where 


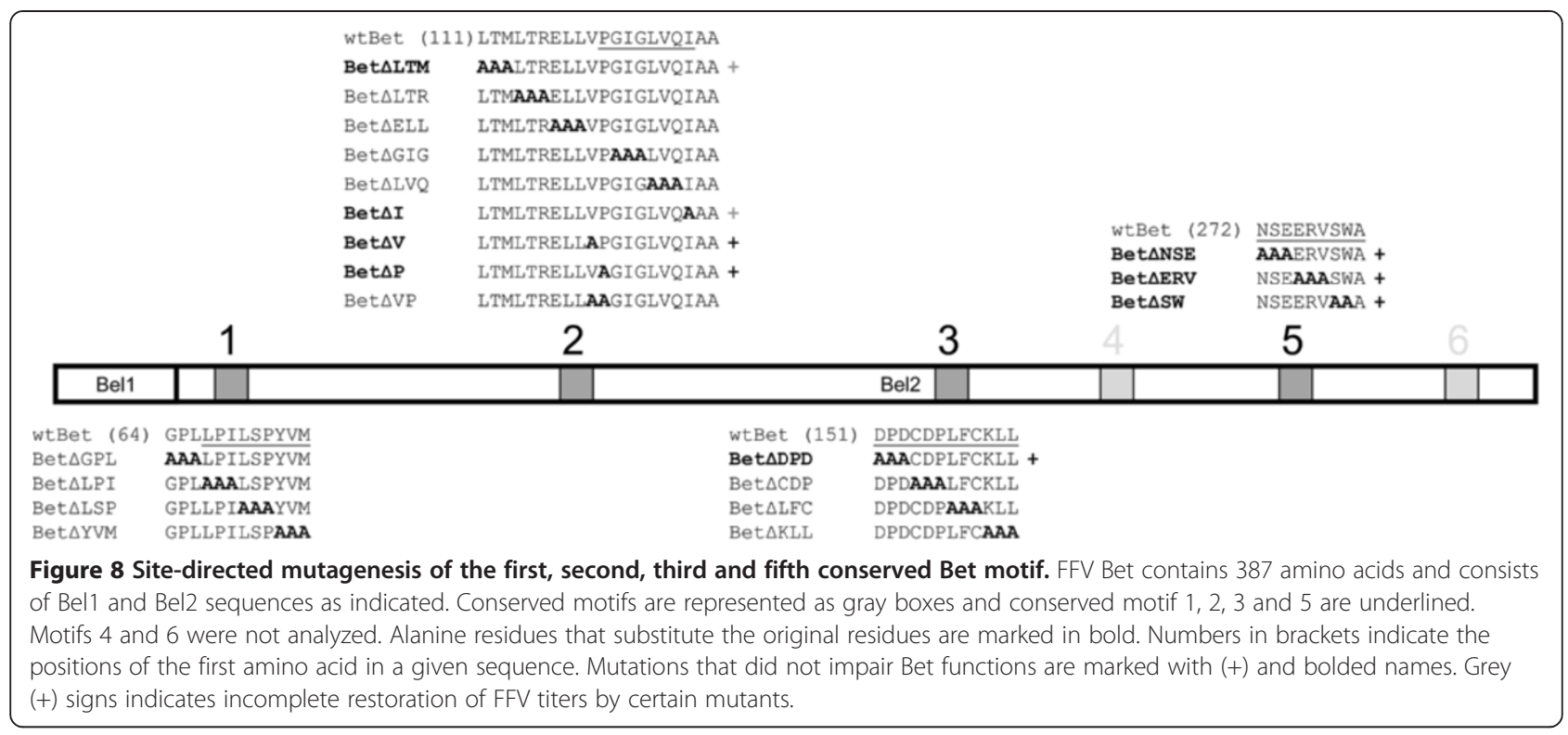

only the last amino acid of motif 2 were exchanged bound to feA3Z2b and partially inactivated this restriction factor. This attenuated phenotype was reproducibly detectable (data not shown). Moreover, Bet $\triangle \mathrm{GPL}$ induced only a minor increase of the FFV titer (Additional file 3C) and it was not pulled down with GST-feA3Z2b (Additional file 4), which may indicate the low sensitivity of the pull down assay used. However, immunoblotting data show that protein expression levels of the Bet mutants were comparable to or slightly lower than wt Bet (Additional file $3 \mathrm{D}, \mathrm{E}$ and F). Therefore, the lack of feA3Z2b counteraction by non-functional Bet mutants (in particular Bet $\Delta \mathrm{GPL}$ ) was not simply the consequence of low protein amounts.

In general, the binding properties of Bet substitution mutants were found to correlate well with their ability to inactivate feA3Z2b, although there were differences in the potential of individual mutants to inactivate feA3Z2b (Additional file 4).

\section{Mutant and wt Bet are localized in the cytoplasm and colocalize with feA3Z2b and feA3Z2a}

Subcellular localization of mutant Bet proteins and their potential colocalization with feA3s were studied by indirect immunofluorescence (IIF) of paraformaldehydefixed HeLa cells using confocal microscopy. Bet was detected with an FFV Bel2-specific serum and is shown in red, while feA3Z2b was labeled with monoclonal anti-HA IgG and is shown in green (Figure 9). Both feA3Z2b-HA and wt Bet predominantly localize to the cytoplasm and, in cells coexpressing both proteins, there is strong colocalization throughout the cytoplasm without any indication of a Bet- or feA3Z2b-mediated relocalization of their corresponding binding partner. This (apparent) colocalization pattern indicates that the wt Bet-A3 complexes do not aggregate in specific regions of the cell but are rather evenly distributed throughout the cytoplasm. Nonfunctional mutant Bet proteins (Bet $\Delta$ LPI, Bet $\triangle \mathrm{CDP}, \mathrm{FFV} / \mathrm{PFV}$ Bet $\mathrm{CH} 4$ ) were, similarly to wt Bet, also evenly distributed in the cytoplasm. In cells coexpressing these nonfunctional Bet mutants and feA3, there are regions of strong colocalization. However, colocalization detected by confocal microscopy does not prove the interaction of the two proteins but indicates that the two proteins have the same spatial occupancy within the cytoplasm. Based on these data, we conclude that the lack of feA3 inactivation by Bet mutants is not the consequence of the physical distance between both proteins.

Since feA3Z2a had been shown in independent studies to localize to the nucleus and the cytoplasm, we analyzed the colocalization of this feA3 isoform with wt Bet and some of the Bet mutants (Figures 10 and 11). In cells transfected only with V5-tagged wt Bet, Bet is predominantly found in the cytoplasm (Figure 10B), while in cells transfected with feA3Z2a, feA3Z2a localizes in both the nucleus and in the cytoplasm (Figure 10A). In most cells that express both wt Bet and feA3Z2a, these two proteins consistently colocalize in both compartments, meaning that Bet is recruited to the nucleus by feA3Z2a (Figure 10C). In addition, in some cells expressing both proteins, feA3Z2a and wt Bet predominantly localized in the cytoplasm (Figure 10D). Functional FFV-PFV Bet $\mathrm{CH} 1$ showed similar colocalization with feA3Z2a as wt 


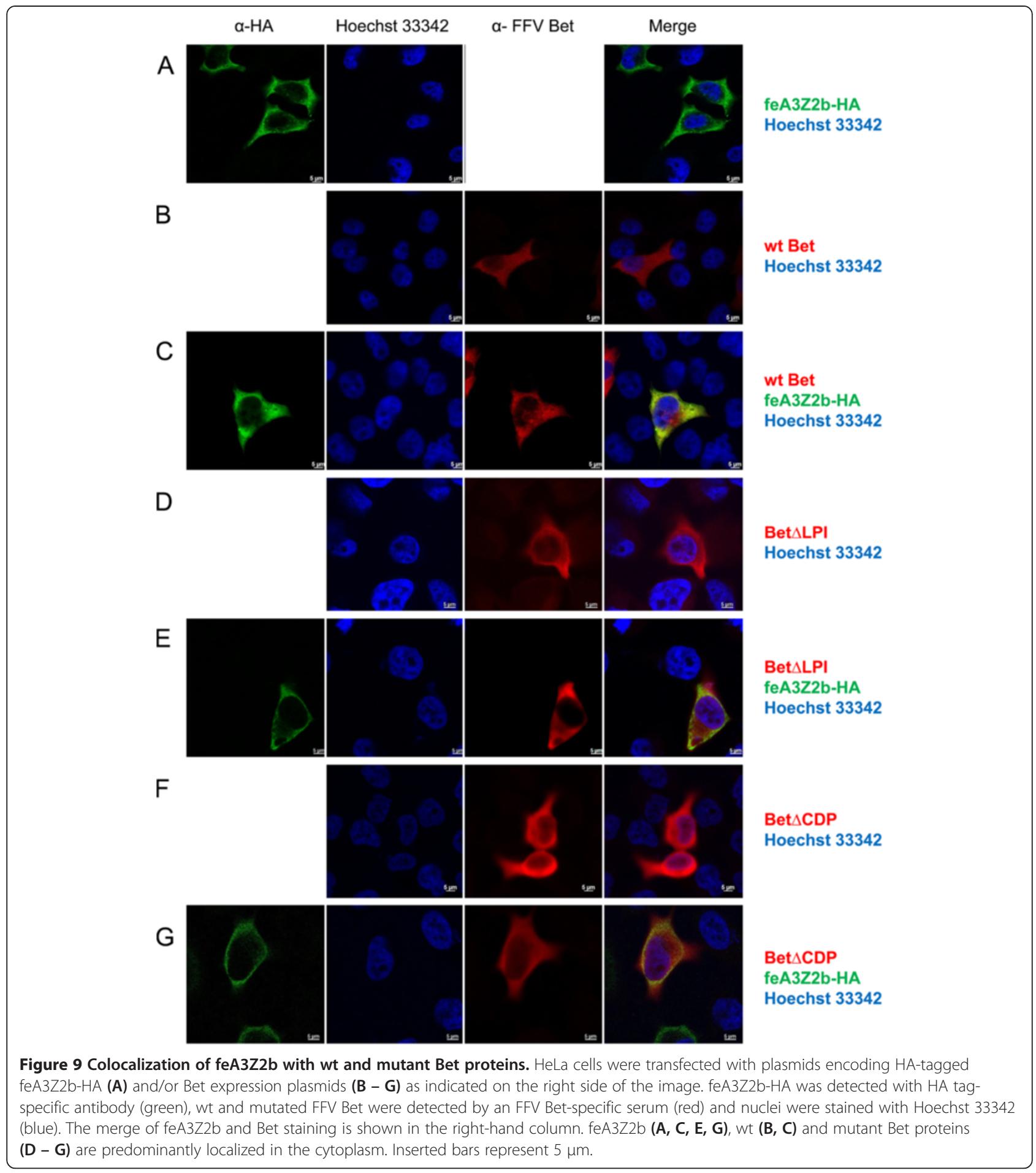

Bet (Figure 10E-H). However, the non-functional Bet mutants tested were not recruited to the nucleus by feA3Z2a (Figure 11).

\section{Discussion}

A3 proteins are cellular restriction factors that deaminate cytidine residues in ssDNA $[8,19,34]$. Two retroviral proteins that counteract A3 restriction factors have been described so far in detail: lentiviral Vif and FV Bet $[8,15,44]$. These two proteins counteract the A3 activity in different ways. While Vif induces proteasomal degradation of A3s [40,59-62], FV Bet does not change the steady state level of A3s $[15,44,47]$. It is assumed that Bet counteracts the activity of A3s by forming stable 


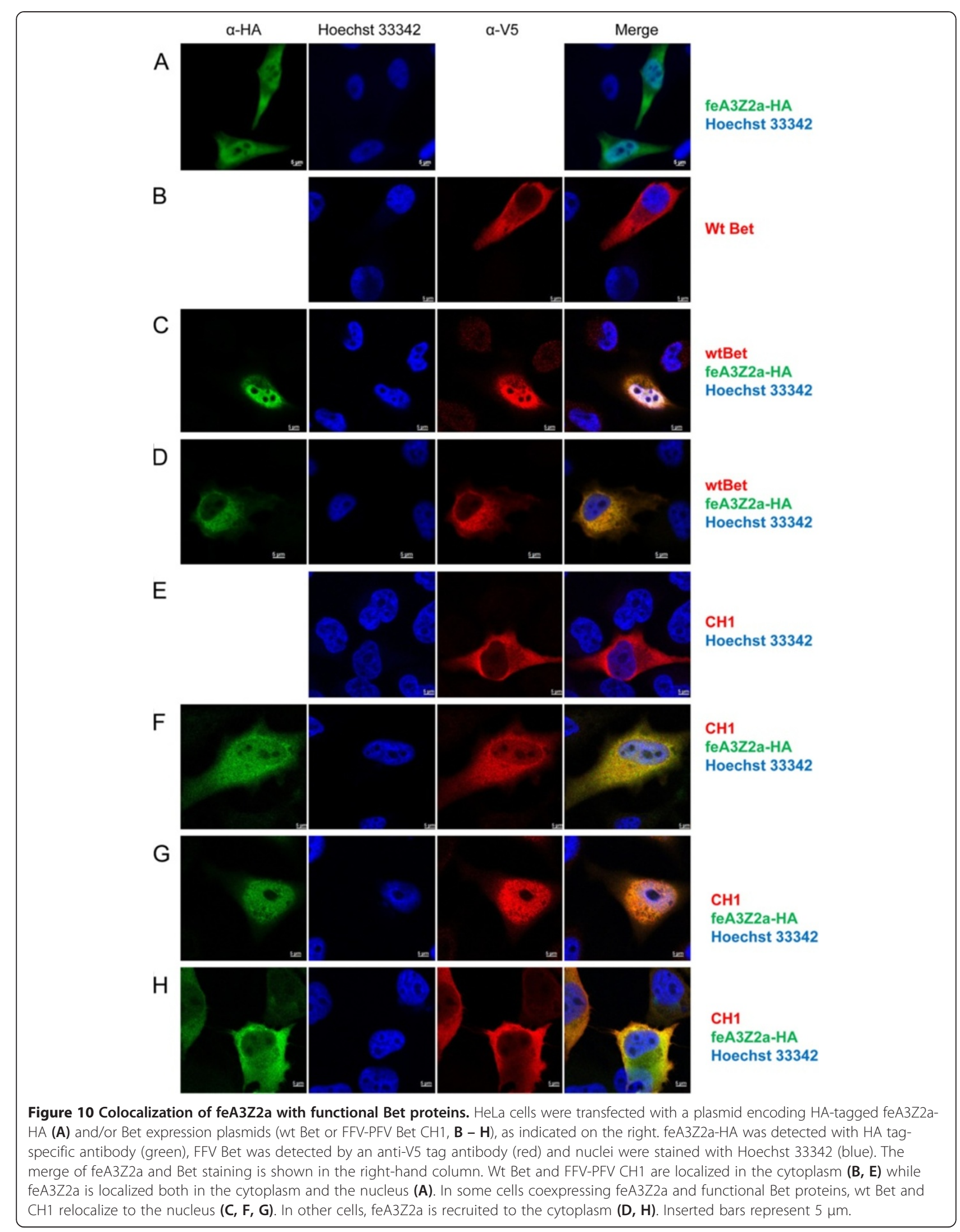




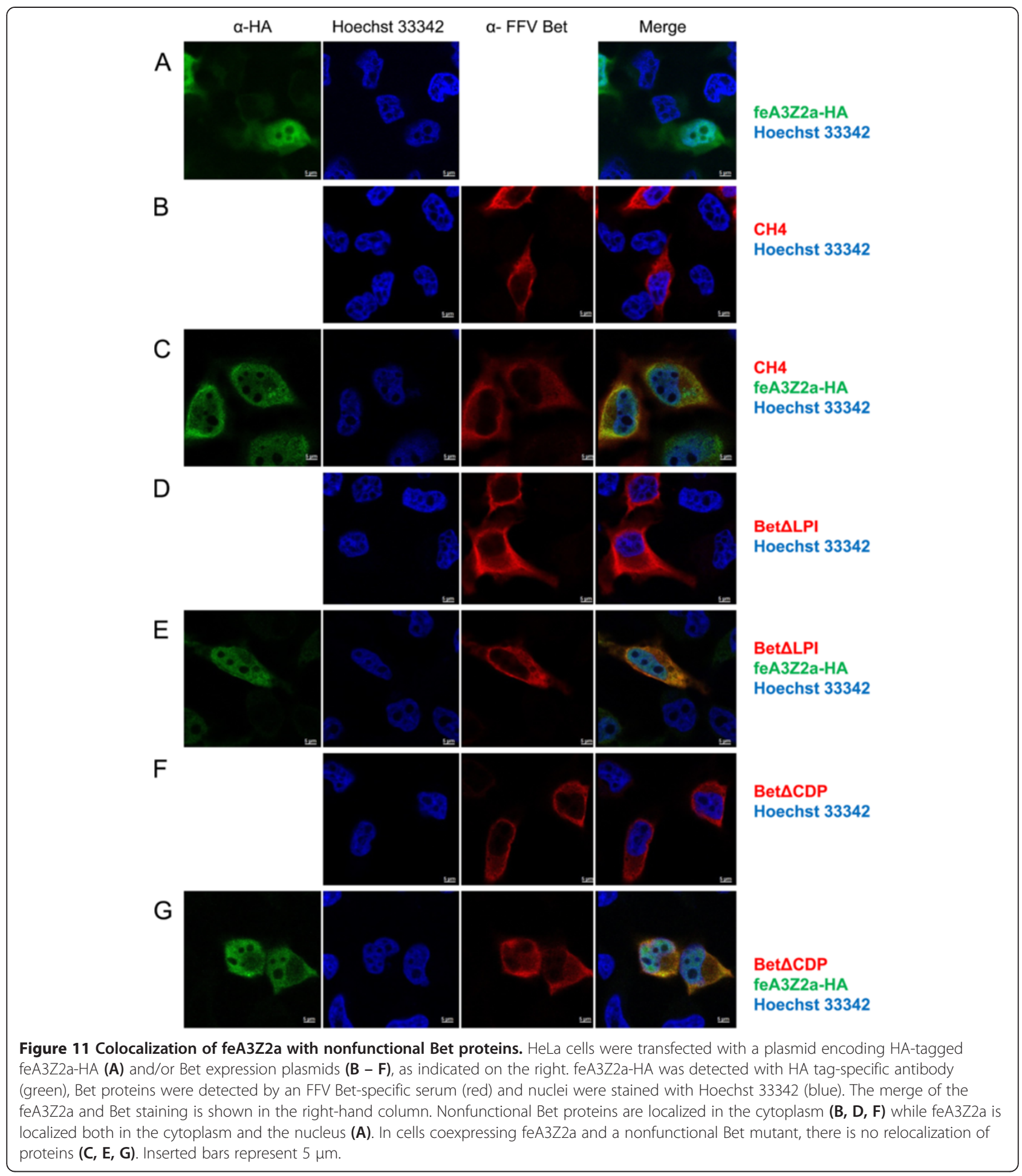

complexes with A3s, thus preventing them from being incorporated into virions $[15,45,47]$.

Our results show that the Bel2 part of FFV Bet protein is essential for species-specific feA3Z2b inactivation and that this domain contains the feA3Z2b interaction site. In contrast, the Bel1 domain increases protein stability but it is not essential for the A3-inactivating function of Bet and can be exchanged between PFV and FFV Bet proteins without detectable loss of function. Alternatively, bel1 RNA sequences or the splice junction may stabilize the transcripts or induce enhanced cytosolic protein biosynthesis. There is no sequence homology 
between PFV and FFV Bel1 (data not shown), leading to the question of whether the presence of any protein domain appended to the N-terminus of Bel2ORF would lead to the same increase of protein stability. However, since higher amounts of Bel2ORF than wt Bet are needed for the same level of A3 counteraction (Figure 2), it might be that the Bel1 domain contributes to anti-A3 activity, for instance, by interacting with some cellular effector protein.

In line with functional and binding studies, bioinformatics showed that the Bel2 parts of all FVs contain conserved motifs. Without knowing the protein structure of Bet, it is difficult to evaluate whether these motifs are localized on the protein surface and may thus directly comprise the feA3 binding site. By molecular modelling using the Robetta full-chain protein structure prediction server $[63,64]$, we obtained hypothetical threedimensional models of Bet. In the most probable model according to QMEAN and ProQservers [65,66], conserved motifs 1, 2 and 3, which have been shown here to be absolutely essential for Bet function, are localized on one side of the Bet protein surface (data not shown). Provided that this model reflects the real Bet structure, the feA3Z2b binding domain may consist of a combination of these motifs. Experimental structure determination of Bet, for instance by crystallography, is required to solve these issues. For instance, it is currently not known whether EFV Bet has a similar structure to the other Bet proteins, since the positions of the second and third conserved motifs are inverted relative to the other known FVs Bet proteins.

Considering the strong coevolution of FVs with their hosts including Bet and A3 proteins $[2,56]$, the binding sites to the species-specific A3 forms may not be conserved between Bet proteins of different FVs. Alternatively, one can imagine that the A3 binding sites may be under highly dynamic, positive evolution. Thus, the A3 binding site may be highly divergent and their primary sequence/structure is likely not maintained. Following this idea, conserved motifs of Bet may be more important for maintaining the tertiary structure of Bet and for proper presentation of the species-specific binding site. The results of this study support both models, since most mutations in these motifs and in flanking sequences impaired the function of FFV Bet.

There is no start codon that would enable expression of the full-length motif 1 to 6 -containing Bel2 protein and there is no conserved ATG start coding in the 5' end of bel2. The first ATG in FFV bel2 is, for instance, already downstream of the first essential motif, and such a Bel2-only protein (Bel2ATG) does not have the capacity to protect against A3 editing, as shown here. Since the bet splice acceptor is located at the 5 'end of the bel2 ORF of all FVs examined [49], Bet expression via a spliced bel1-bel2 fusion transcript allows expression of all conserved bel 2 motifs. This mode of expression may be an efficient way to generate high amounts of Bet, since the internal promoter (IP) is very active upon full transactivation [49]. In addition, Bel1/Tas and Bet expression via alternative splicing of a single transcription unit may be an important regulatory mechanism of FV replication. Through regulated splicing of IP transcripts, early expression of Bel1/Tas may initiate and maintain high levels of viral gene expression, while later expression of Bet protects the viral genome from A3 editing during and after particle formation and reverse transcription. Expression of Bet during the late phase of replication and progeny virus production may therefore be the result of FV evolution to avoid A3 incorporation into virus particles during replication in A3-positive cells. It may be even more important for FVs due to the fact that reverse transcription may already occur in the virus-producing cells [52].

For all tested mutants, binding to feA3 correlated with the inactivation of this restriction factor. The tight association between feA3 binding and inactivation by FFV Bet supports the hypothesis that Bet inactivates A3s by creating strong complexes $[15,45]$. This mechanism of A3 inactivation requires high amounts of Bet proteins and may be the reason for high expression levels of Bet in FV-infected cells and animals $[48,67,68]$. In line with this, results of this study show that, for efficient inactivation of A3s, the amount of Bet must be at a sufficiently high level; small to modest amounts of Bet do not or only partially counteract A3 restriction.

Hypothetically, binding of Bet to feA3Z2b could mask the Gag binding site on feA3Z2b, thus preventing its incorporation into viral particles. Results presented here indicate that almost the whole Bel2ORF is important for feA3Z2b binding and suggest that Bel2ORF may wrap around feA3Z2b and prevent its interaction with Gag. Alternatively, binding of Bet to A3s could block transport of A3 into viral particles or interfere with some cellular factor(s) [15] crucial for A3 packaging into viral particles.

Confocal microscopy indicates that both Bet and feA3Z2b are apparently uniformly distributed in the cytoplasm and thus do not allow detection of protein relocalization upon complex formation. In contrast, feA3Z2a, which also restricts FFV and is inhibited by Bet binding $[33,47]$, is also present in the nucleus. Wt Bet and functional Bet mutants colocalize to the nucleus in the presence of this restriction factor. Surprisingly, in some cells, feA3Z2a seems to retarget Bet which is somewhat counterintuitive since the smaller protein dictates sub-cellular localization of its larger binding partner. Why not all cells show this phenotype remains to be determined.

Bet is a highly expressed viral protein and a diagnostic marker for FV infection [51,67]. All known FVs express 
Bet, strongly supporting the importance of this protein for efficient FV replication. Sequences corresponding to bet/bel2, as determined by localization and sequence homology, have also been identified in the SloEFV, active more than 100 million years ago [43], indicating that Bet is an ancient gene. In addition, a sequence corresponding to bel2 is even present in coelacanth endogenous FV [50], though it contains only the first conserved motif (data not shown). This may be a result of the accumulation of mutations from the lack of selective pressure leading to truncations of the parental bel2 ORF.

Although Bet and Vif counteract A3 restriction factors, their localization in viral genomes and their fundamentally different mechanisms of A3 counteraction suggest that these two proteins may have evolved independently from each other. The ancestral protein(s) of Vif and Bet may have been cellular A3 binding proteins with regulatory functions. In this model, there was no need for high level of vif expression in lentiviruses, since Vif acts as an adaptor for a catalytic degradation of A3 proteins. In contrast, the internal promoter (IP) of FVs provides high levels of Bet for efficient inactivation of A3s simply by binding. Alternatively, FVs might have developed an IP to increase the expression of Bet, since Bet does not recruit the cellular degradation machinery.

\section{Conclusions}

The Bel2 domain of FFV Bet contains conserved motifs and is essential for inactivation of feA3s. Although the Bel1 part is not directly involved in binding and inactivation of these restriction factors, it is important for expression of full-length Bel2ORF and protein stability. The absolute correlation between binding and inactivation of feA3Z2b by Bet mutants suggests that Bet inactivates A3s simply by creating strong complexes. Such a strong feA3Z2b-Bet binding is most probably a result of a very long coevolution of these two proteins. Considering that sloth and coelacanth FVs contain sequences that correspond to bet $[43,50]$ and its conserved motifs, it is possible that this coevolution between Bet and A3s or their progenitors extends far back in vertebrate evolution.

\section{Methods}

\section{Cell culture and virological methods}

HEK293T and FeFab cells were propagated as described before [69]. HEK293T cells were seeded in $6 \mathrm{~cm}$ or $10 \mathrm{~cm}$ dishes and transfected using a modified calcium phosphate method [69]. FFV titers were determined by a $\beta$-galactosidase assay using FFV-FAB (FeFab) cells grown in 96-well plates [69]. FFV-containing supernatants were serially diluted 1:5, titrations were done in triplicate.

\section{Plasmids and DNA transfection}

pFeFV-BBtr and pcfeA3 plasmids have been already described $[33,51,69]$. The bacterial expression vector for glutathione-S-transferase (GST)-tagged feA3Z2b has been described recently [47].

\section{Molecular cloning of FFV Bet mutants}

To construct Bet N-terminal deletion mutants, the corresponding bel2 sequences were amplified by Bel2ORF, Bel2ATG, FFVBet $\Delta$ N82, or FFVBet $\Delta$ N92 sense primers (containing a HindIII site) and FFVbel2as antisense primer using plasmid pBC-FFV-Bet as template [51]. All reactions were performed with Phusion ${ }^{\mathrm{Tm}}$ High-Fidelity DNA Polymerase (NEB, Germany). Blunt-ended products were digested with HindIII and inserted into HindIII/SmaI-digested pBC12CMV.

To generate a V5-tagged Bet, bet was amplified with Bet sense and Bet-V5 antisense primers as above and cloned into HindIII/SmaI-digested pBC12CMV. To introduce V5 into Bet N-terminal deletion mutants, AccI/XmaI fragments of pBC-Bel2ORF, pBC-Bel2ATG, pBC-Bet $\Delta \mathrm{N} 82$ and $\mathrm{pBC}$-Bet $\triangle \mathrm{N} 92$ were replaced by the corresponding fragment of pBC-FFVBetV5.

V5-tagged C-terminal Bet deletion mutants were constructed by amplification of corresponding bet sequences with Bet sense and one of three different antisense primers (dC1-V5, dC2-V5 or dC3-V5, Table 1) with a V5 epitope. PCR products were cloned into HindIII/SmaIdigested pBC12CMV.

To construct chimeric Bet proteins, corresponding parts of PFV and FFV bet were fused in-frame by fusion PCR [70]. Corresponding parts of each gene were amplified in individual PCR reactions. For instance, for cloning of FFV-PFV CH1, PCR1 with chimera-s1 and ch1-as using $\mathrm{pBC}$-FFV-Bet as template and PCR2 with ch1-s2 and Bet V5 as primers and pBC-PFV-Bet as template were performed as given above. Finally, the amplicons were fused in PCR3 using chimera-s1 and Bet-V5 antisense primers. Blunt-ended PCR products were digested with HindIII and cloned into HindIII/SmaI-digested pBC12CMV. To construct FFV-PFV CH7 to CH11, FFV Bet was amplified with generic ch-sense1-bspe1 and a mutant-specific as1 primer. PFV Bet was amplified with the corresponding s2 primer and generic chimera-as primer. Products of both PCRs were fused in a third PCR reaction with ch-sense1-bspeI and chimera-as primers. Final products were cloned into BspEI/SmaI digested pBC-Bet-V5.

Alanine scanning mutagenesis of the second conserved motif of FFV Bet and flanking residues was performed by amplifying bet between the unique HindIII and KpnI sites using Bet-sense and one of three antisense primers (BetAlaRI, BetAlaRII, BetAlaRIII for $\triangle \mathrm{ELL}, \triangle \mathrm{LTR}$ and $\triangle \mathrm{LTM}$, respectively). Products were cloned into HindIII/ KpnI-digested pBC-Bet. Residues downstream of KpnI 
Table 1 Primers used for cloning and site-directed mutagenesis

\begin{tabular}{|c|c|}
\hline Name & Sequence (5'-3' direction) \\
\hline \multicolumn{2}{|c|}{ Cloning of $\mathrm{N}$-terminal deletion mutants of FFV Bet } \\
\hline FFVbel2ORF & ATCCCAAGCTTGCCACCATGGTCGGAAAGAATCCGGAAC (HindIII) \\
\hline FFVbel2ATG & ATCCCAAGCTTGCCACCATGGCTTGGGACAACCCTC (HindIII) \\
\hline FFVBet $\Delta N 82$ & ATATCCCAAGCTTGCCACCATGGTGGTCACACGTCTGGTG (HindIII) \\
\hline FFVBet $\triangle N 92$ & ATATCCCAAGCTTGCCACCATGGAATCATGGAAGAAGTATC (HindIII) \\
\hline FFVbel2as & GGGTCACTCGAGCTATTCAGAGTCAGATGACTC (Smal) \\
\hline \multicolumn{2}{|c|}{ Cloning of V 5 tagged FFV Bet and C-terminal deletion mutants of Bet } \\
\hline Bet-sense & CTCCCCTCGAAGCTITCTGGGATATGTAAAACC (HindIII) \\
\hline $\mathrm{dC} 1-\mathrm{V} 5$ & GGGTCAGGTGCTGTCCAGGCCCAGCAGGGGGTTGGGGATGGGCTTGCCATCATCAGCTTGTGC \\
\hline$d C 2-V 5$ & GGGTCAGGTGCTGTCCAGGCCCAGCAGGGGGTTGGGGATGGGCTTGCCCAGCAGAGAGTATTC \\
\hline$d C 3-V 5$ & GGGTCAGGTGCTGTCCAGGCCCAGCAGGGGGTTGGGGATGGGCTTGCCTCTTCCATCAGGAAG \\
\hline Bet-V5 antisense & GGGTCAGGTGCTGTCCAGGCCCAGCAGGGGGTTGGGGATGGGCTTGCCTTCAGAGTCAGATGA \\
\hline \multicolumn{2}{|c|}{ Alanine scanning mutgenesis } \\
\hline BetAlaRl & ATGCAAGATGATGGTACCGCAGCGGCTCTAGTTAGCATAGTCAAATC (Kpnl) \\
\hline BetAlaRII & ATGCAAGATGATGGTACCAACAATTCGGCAGCGGCCATAGTCAAATCCCTCTC (Kpnl) \\
\hline BetAlaRIII & ATGCAAGATGATGGTACCACAATTCTCTAGTTAGGGCAGCGGCATCCCTCTCCCCACAATC (Kpnl) \\
\hline BetAlaFI & ATGCAAGATGATGGTACCAGCCGCTGCCCTGGTACAAATCGCCGCTAC (Kpnl) \\
\hline BetAlaFIl & ATGCAAGATGATGGTACCAGGAATAGGCGCCGCTGCCATCGCCGCTACACTTAC (Kpnl) \\
\hline BetAlaFIII & ATGCAAGATGATGGTACCAGGAATAGGCCTGGTACAAGCCGCCGCTACACTTACTAAAACC (Kpnl) \\
\hline BetAlaR2 & AGGTAAAAGATTCCTATGTCTACGCACAATC (ACCl) \\
\hline R-Xhol & ATGCAAGATGATGGTACCAACAATTCTCGAGTTAGCATAGTC (Kpnl) \\
\hline ForwardVP & 5'ATGCAAGATGATCTCGAGAATTGTTGGCTGCCGGAATAGGCCTGGTAC (Xhol) \\
\hline ForwardV & 5'ATGCAAGATGATCTCGAGAATTGTTGGCCCCAGGAATAGGCCTGGTAC (Xhol) \\
\hline ForwardP & ATGCAAGATGATCTCGAGAATTGTTGGTAGCCGGAATAGGCCTGGTAC (Xhol) \\
\hline dGPL-AS1 & TGGAAGGGCAGCGGCGGGAACATCCTGCTTCTTG \\
\hline dGPL-S2 & GCCGCTGCCCTTCCAATTCTGAGTCCG \\
\hline dLPI-AS1 & ACTCAGGGCAGCGGCGAGTGGGCCGGGAACATCC \\
\hline dLPI-S2 & GCCGCTGCCCTGAGTCCGTATGTAATGG \\
\hline dLSP-AS1 & TACATAGGCAGCGGCAATTGGAAGGAGTGG \\
\hline dLSP-S2 & GCCGCTGCCTATGTAATGGCTTGGGACAACC \\
\hline dYVM-AS1 & CCAAGCGGCAGCGGCCGGACTCAGAATTGGAAG \\
\hline dYVM-S2 & GCCGCTGCCGCTTGGGACAACCCTCAG \\
\hline dDPD-AS1 & ATCACAGGCAGCGGCGGTTCTAGAACCTGTAATAC \\
\hline dDPD-S2 & GCCGCTGCCTGTGATCCTTTGTTCTGTAAG \\
\hline dCDP-AS1 & GAACAAGGCAGCGGCATCTGGGTCGGTTCTAGAAC \\
\hline dCDP-S2 & GCCGCTGCCTTGTTCTGTAAGTTGTTATGC \\
\hline dLFC-AS1 & CAACTTGGCAGCGGCAGGATCACAATCTGGGTCGGTTC \\
\hline dLFC-S2 & 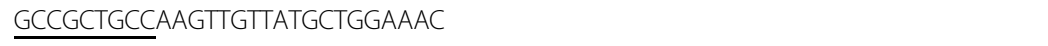 \\
\hline dKLL-AS1 & CCAGCAGGCAGCGGCACAGAACAAAGGATCACAATC \\
\hline dKLL-S2 & GCCGCTGCCTGCTGGAAACAAAATATAC \\
\hline dNSE-AS1 & CCTCTCGGCAGCGGCCCCAGAGGCACTTCCAAATATG \\
\hline dNSE-S2 & GCCGCTGCCGAGAGGGTGTCATGGGCCAAAG \\
\hline dERV-AS1 & CCATGAGGCAGCGGCCTCACTGTTCCCAGAGGCAC \\
\hline dERV-S2 & GCCGCTGCCTCATGGGCCAAAGAGAATTC \\
\hline
\end{tabular}


Table 1 Primers used for cloning and site-directed mutagenesis (Continued)

\begin{tabular}{|c|c|}
\hline dSW-AS1 & СTCTTIGGCAGCGGCCACCCTCTCCTCACTGTTCC \\
\hline dSW-S2 & GCCGCTGCCAAAGAGAATTCTCACAGAG \\
\hline AS2-Xmal & AGTGTAAGTTCACCCGGGTCACTCGAGCTATTCAGAGTCAGATGACTC (Smal, Xmal) \\
\hline \multicolumn{2}{|c|}{ Cloning of chimeric FFV/PFV Bet fusion proteins } \\
\hline chimera-s1 & ATGCAAGATGATAAGCTITAGCTGCAGCAACAAAG (HindIII) \\
\hline ch1-as1 & GTGTTCCGGATTCTITCCAGCAATAGTCAGCTCTCC \\
\hline ch1-s2 & GGAGAGCTGACTATTGCTGGAAAGAATCCGGAACAC \\
\hline ch2-as1 & GTGTTCCGGATTCTTTCCCTTCTGAGCAATCATTTC \\
\hline ch2-s2 & GAAATGATTGCTCAGAAGGGAAAGAATCCGGAACAC \\
\hline ch4-as1 & CCACGTGTGACCACGTTTGCATAGTGATCCTGGCTC \\
\hline ch4-s2 & GAGCCAGGATCACTATGCAAACGTGGTCACACGTGG \\
\hline ch5-as1 & GTAAGTGTAGCGGCGATCTGAATGTTCACCTGACC \\
\hline ch5-s2 & GGTCAGGTGAACATTCAGATCGCCGCTACACTTAC \\
\hline ch6-as1 & CAGGCACCATTCTTCTAGTTGCTITTGGCCCATTGC \\
\hline ch6-s2 & GCAATGGGCCAAAAGCAACTAGAAGAATGGTGCCTG \\
\hline ch3-as1 & GTGTTCCGGATTCTTTCCCATTTCCTCTGGTGTGGGGATCC \\
\hline ch3-s2 & GGATCCCCACACCAGAGGAAATGGGAAAGAATCCGGAACAC \\
\hline ch-sense1- bspei & ATGCAAGATGATTCCGGAACACCCAAGACGGATC (BspEI) \\
\hline ch7-as1 & GATTCCAAAGAGGGTTGGCTGAGGGTTGTCCCAAGC \\
\hline ch7-s2 & GCTTGGGACAACCCTCAGCCAACCCTCTTTGGAATC \\
\hline ch8-as1 & CTGATAATTCTTATAAAATTGTACCAGGCCTATTCC \\
\hline ch8-s2 & GGAATAGGCCTGGTACAATTITATAAGAATTATCAG \\
\hline ch9-as1 & GTCTGCACAGCCAGGTTTTTGGTTACACTCTCTAGGGTC \\
\hline ch9-s2 & GACCCTAGAGAGTGTAACCAAAAAACCTGGCTGTGCAGAC \\
\hline ch10-as1 & AATCCTAGGATTGGTGAAGACTCTGGGATACAGGGAAGC \\
\hline ch10-s2 & GCTTCCCTGTATCCCAGAGTCTTCACCAATCCTAGGATT \\
\hline ch11-as1 & CTGTCAATGTTCTGATCTTCTCAGGATCACAGGCTATG \\
\hline ch1-s2 & CATAGCCTGTGATCCTGAGAAGATCAGAACATTGACAG \\
\hline chimera-as & GGGTCAGGTGCTGTCCAGGCCCAGCAGGGGGTTGGGGATGGGCTTGCCGAAGGGTCCATCTGAGTC (Smal) \\
\hline
\end{tabular}

All primer sequences are in the $5^{\prime}$ to $3^{\prime}$ orientation. Sequences in italics correspond to restriction enzyme recognition sites that were used for cloning; the names of the enzymes are given in brackets. Underlined sequences mark the mutations introduced. Sequences encoding the V5 tag are given in bold-face letters.

were mutated by amplifying bet from KpnI to AccI using sense primers (BetAlaFI, BetAlaFII and BetAlaFIII for $\Delta \mathrm{GIG}, \triangle \mathrm{LVQ}$ and $\Delta \mathrm{I}$, respectively) and BetAlaR2 as general antisense primer. Products were cloned into $\mathrm{pBC}$ Bet with KpnI and AccI. Using V, P, or VP sense primers, the BetAlaR antisense primer, and Bet-XhoI (in which an XhoI site was silently introduced with Bet sense and RXhoI antisense primer) as template, corresponding parts of Bet were amplified and the indicated codons exchanged by alanine codons. PCR products were cloned into pBC-Bet-XhoI using XhoI and AccI.

Residues in the first, third and fifth conserved motifs were exchanged by fusion PCR. Bet was amplified with Bet-sense and AS1 antisense primers. The second PCR was performed with S2 sense primers and AS-XmaI primer, covering the bet stop codon and carrying an XmaI site (e. g. fusion PCR with Bet-sense, dGPL-AS1, dGPL-S2, AS-XmaI primers for $\triangle G P L)$. The two fragments were fused by PCR using Bet-sense and AS-XmaI. The final product was cloned into $\mathrm{pBC} 12-\mathrm{CMV}$ using HindIII and XmaI.

\section{Coimmunoprecipitation (co-IP) and immunoblotting}

To study the interaction between proteins expressed in eukaryotic cells, HEK293T cells were seeded in $10 \mathrm{~cm}$ dishes and transfected with $6 \mu \mathrm{g}$ of pcfeA3Z2b-HA and $12 \mu \mathrm{g}$ of PFV Bet, FFV Bet, or chimeric FFV/PFV Bet expression plasmid. Two d.p.t., cells were lysed in TLB (20 mM Tris, pH 7.4, $137 \mathrm{mM} \mathrm{NaCl}, 10 \%$ glycerol, $2 \mathrm{mM}$ EDTA, 1\% Triton X-100 and protease inhibitor). Lysates were cleared by $5 \mathrm{~min}$ centrifugation at $500 \times \mathrm{g}$. $100 \mu \mathrm{l}$ of the cell lysate was incubated with monoclonal mouse anti- 
HA IgG (Abcam, Cambridge, UK) and protein-G-sepharose overnight at $4^{\circ} \mathrm{C}$. The beads were washed three times in TLB. After the last wash, beads were boiled in sample buffer and precipitated proteins were detected by immunoblotting. feA3Z2b-HA was detected with monoclonal anti-HA IgG (Abcam). PFV Bet was detected with PFV Bet-specific serum. Wt FFV Bet and chimeric proteins were detected with monoclonal mouse anti-V5 IgG (Sigma-Aldrich, Munich, Germany). Membranes were incubated with horseradish peroxidase-conjugated secondary antibodies (Sigma-Aldrich) and visualized by enhanced chemiluminescence (ECL, GE Healthcare, Freiburg, Germany). Densitometry was performed of scanned autoradiograms using the ImageJ software [71].

\section{Immunofluorescence and confocal microscopy}

HeLa cells were grown on coverslips in 6-well plates and transfected with $1 \mu \mathrm{g}$ feA3Z2b and feA3Z2a expression plasmids and $2 \mu \mathrm{g}$ of plasmid expressing wt or mutant Bet. 2 d.p.t., cells were fixed with $3 \%$ paraformaldehyde in PBS for $15 \mathrm{~min}$. and permeabelized with $0.1 \%$ TritonX-100 for $7 \mathrm{~min}$. Cells were incubated with rabbit FFV Bet-specific serum (1:1000) and monoclonal mouse antiHA IgG (Abcam) diluted in 3\% BSA in PBS. Alexa Fluor ${ }^{\circ}$ 488 goat anti-mouse IgG and Alexa Fluor 594 goat antirabbit IgG (both from Invitrogen, Karlsruhe, Germany) were used as secondary antibodies in a dilution of 1:2000. Nuclei were stained with Hoechst 33342 (1:2000). Cells were imaged using a Zeiss LSM700 confocal microscope (Zeiss, Jena, Germany).

\section{Protein pulldown assays}

For pull down assays of the feA3 with FFV-Bet, HEK293T cells were transfected with $10 \mu \mathrm{g}$ of FFV Bet expression plasmids. Two d.p.t., cells were lysed in $250 \mu \mathrm{l}$ TLB and lysates were cleared by centrifugation. $100 \mu \mathrm{l}$ of cleared supernatants were incubated with approximately one mg GST-feA3Z2b fusion proteins purified by glutathioneS-sepharose affinity chromatography according to the manufacturer's instruction (GE Healthcare, UK) as described previously [47]. Samples were incubated overnight at $4^{\circ} \mathrm{C}$ and washed three times with TLB. As negative control, samples were incubated with GST alone. Samples were boiled in sample loading buffer and separated and probed by SDS-PAGE and immunoblotting.

\section{Bioinformatics}

Conserved motives in Bet were identified using MEME (Multiple Expectation maximization for Motif Elicitation [57]). MEME is an unsupervised learning algorithm for discovering motifs in sets of protein or DNA sequences that quantifies/predicts the chance of interchangeability of defined residues in related sequences [57].
DNA and protein sequence analysis were performed using Geneious and ClustalW2. Molecular models of FV Bet were obtained using the Robetta Protein Prediction Server (http://robetta.bakerlab.org) [63,64]. Predicted models were evaluated using the QMEAN and ProQ servers $[65,66]$.

\section{Additional files}

Additional file 1: N-terminal deletion mutants are detected to similar degrees by the Bet-specific serum and a V5 tag-specific antibody. HEK293T cells were transfected with $5 \mu \mathrm{g}$ of pBC-Bet-V5, pBCBel2ORF-V5, pBC-Bel2ATG-V5, pBC-Bet $\triangle \mathrm{N} 82-\mathrm{V} 5$ or pBC-Bet $\triangle \mathrm{N} 92-\mathrm{V} 5$ and harvested 2 d. p.t. $40 \mu \mathrm{g}$ of proteins from each cell lysate was used for protein detection. Two SDS gels were used for immunoblotting and one membrane was incubated with the FFV Bet-specific serum (A) and the other with the $\mathbf{V} 5$-specific antibody (B). The wt and mutant Bet proteins were detected with similar efficacy using both Bet-specific serum and V5 tag-specific antibody.

Additional file 2: C-terminal Bet deletion mutants do not counteract feA3Z2b-mediated restriction. (A) Schematic presentation of full-length Bet and C-terminal Bet deletion mutants. Grey boxes represent conserved motifs and green boxes represent $V 5$ tags. (B) HEK293T cells were cotransfected with $4 \mu \mathrm{g}$ of pCF-BBtr and pCDNA or pfeA3Z2b-HA and $5 \mu \mathrm{g}$ of plasmids expressing wt Bet or Bet C-terminal deletion mutants, as indicated. One d.p.t., ALLN $(25 \mu \mathrm{M})$ or DMSO was added to the cells. Two d.p. t., titration was performed in triplicate and mean titer values are presented. Error bars represent standard deviations. Labels below the columns indicate the clone that was cotransfected. The line above the columns indicates the presence of feA3Z2b. Bet and Bet$\checkmark 5$ efficiently restored viral titer in the presence of ALLN. C-terminal Bet deletion mutants did not restore viral titer, although expression levels increased in the presence of ALLN. (C) $40 \mu \mathrm{g}$ of proteins from each cell lysate were used for immunoblot analysis. Wt and mutant Bet were detected either with V5-specific antibody or an FFV Bet serum. Levels of C-terminal deletion mutants partially increased in the presence of ALLN, while levels of the other proteins were unchanged. HA tag-specific antibody was used for feA3Z2b-HA detection, an FFV MA serum for Gag detection, and detection of $\beta$-actin confirmed proper loading of the samples. (D) HEK293T cells were transfected with $10 \mu \mathrm{g}$ of pBC-Bet $\triangle$ C244-V5, pBC-Bet $\triangle C 292-V 5$, pBCBet $\triangle$ C357-V5 or pBC-Bet-V5. Protein expression increased by supplementing cell culture medium with $8 \mathrm{mM}$ sodium butyrate. Two d.p.t., cells were lysed and incubated with affinitypurified GST or GST-feA3Z2b. Pulled down proteins were detected by immunoblotting with V5-specific antibody. Hatched lines mark empty gel lanes to separate individual assays. Only full-length Bet-V5 was pulled down with GST-feA3Z2b. The presence $(+)$ or the absence $(-)$ of GST and GST-A3Z2b are indicated; $C L$, cell lysate.

Additional file 3: Site-directed mutagenesis of conserved FFV Bet motifs 1 to 3 impair Bet function. HEK293T cells were cotransfected with pCF-BBtr and pcDNA or pfeA3Z2b and plasmid expressing wt or mutant FFV Bet proteins as indicated. (A, B, C) Two d.p.t., titration was performed in triplicate using FeFab cells and mean values are

represented; error bars represent standard deviation. The line above the graph indicates the presence of feA3Z2b. Black dots on white bars indicate motif 1 mutants; hatched bars, motif 2 mutants; striped bars, motif 3 mutants; white dots on black bars, motif 5 mutants; black bars, wt Bet; white bars, pcDNA (see also Figure 8). In the presence of feA3Z2b, the FFV-BBtr titer decreased 3 to 4 logs. FFV Bet, used as a positive control restored the viral titer in all cases. None of the Bet proteins with mutations in the first conserved motif were functionally active, although there were minor variations of the titer. Bet $\triangle D P D$ is the only mutant with substitutions in the third conserved motif that was functionally active. All mutants with substituted amino acids in the fifth conserved motif counteracted feA3Z2b-mediated FFV-BBtr restriction with slightly reduced efficacies (D, E, and F). $40 \mu \mathrm{g}$ of proteins from each cell lysate were used for immunoblot analysis. Wt and mutant Bet 
proteins were detected with FFV Bet-specific serum. HA tag-specific antibody was used for feA3Z2b-HA detection, MA serum for Gag detection, and $\beta$-actin as a loading control.

Additional file 4: Bet proteins with mutations in conserved motifs 1-3 cannot bind feA3Z2b. HEK293T cells were transfected with $10 \mu \mathrm{g}$ of wt or mutant Bet expression plasmids. Two d.p.t., cells were lysed and incubated with affinity-purified GST or GST-feA3Z2b. After overnight incubation, pulled down proteins were detected by immunoblotting with FFV Bet-specific serum. Hatched lines mark empty gel lanes to separate individual pulldown assays. The presence (+) or the absence (-) of GST and GST-A3Z2b are indicated. The upper panel shows pull down assays performed with Bet mutants carrying mutations in the second conserved motif. Pulldown assays with mutations in the first, third or fifth conserved motif of Bet are boxed.

\section{Competing interests}

The authors declare no conflict of interests.

\section{Authors' contributions}

DSL designed and performed experiments. DSL and ML wrote the manuscript. JL and AMR contributed to data evaluation and helped in writing of manuscript. AHW, MM and JD performed bioinformatics and structural modeling of Bet.CM provided unpublished data on feA3Z2a nuclear localization and provided reagents and controls. ML designed and supervised the study. All authors read and approved the final manuscript.

\section{Acknowledgements}

We thank Lutz Gissmann for continuous support and the DKFZ Light Microscopy Facility for guidance and help. DSL was supported by the DKFZ PhD Program and CM is supported by the Heinz-Ansmann Foundation for AIDS research. JD and ML are supported by a grant from the VolkswagenStiftung.

\section{Author details}

'Research Program Infection and Cancer, Department Genome Modifications and Carcinogenesis, German Cancer Research Center (DKFZ), Heidelberg, Germany. ${ }^{2}$ Core Facility Genomics and Proteomics, German Cancer Research Center (DKFZ), Heidelberg, Germany. ${ }^{3}$ Robert Koch-Institute, Center for HIV and Retroviruses, Berlin, Germany. ${ }^{4}$ Clinic for Gasteroenterology, Hepatology and Infectology, Medical Faculty, Heinrich-Heine-University, Düsseldorf, Germany.

Received: 15 February 2013 Accepted: 19 July 2013

Published: 24 July 2013

\section{References}

1. Harris RS, Hultquist JF, Evans DT: The restriction factors of human immunodeficiency virus. J Biol Chem 2012, 287:40875-40883.

2. Münk C, Hechler T, Chareza S, Löchelt M: Restriction of feline retroviruses: lessons from cat APOBEC3 cytidine deaminases and TRIM5alpha proteins. Vet Immunol Immunopathol 2010, 134:14-24.

3. Peng G, Lei K, Jin W, Greenwell-Wild T, Wahl SM: Induction of APOBEC3 family proteins, a defensive maneuver underlying interferon-induced anti-HIV-1 activity. J Exp Med 2006, 203:41-46.

4. Liberatore RA, Bieniasz PD: Tetherin is a key effector of the antiretroviral activity of type I interferon in vitro and in vivo. Proc Natl Acad Sci USA 2011, 108:18097-18101.

5. Li N, Zhang W, Cao X: Identification of human homologue of mouse IFNgamma induced protein from human dendritic cells. Immunol Lett 2000, 74:221-224.

6. Stopak KS, Chiu YL, Kropp J, Grant RM, Greene WC: Distinct patterns of cytokine regulation of APOBEC3G expression and activity in primary lymphocytes, macrophages, and dendritic cells. J Biol Chem 2007, 282:3539-3546.

7. Carthagena L, Bergamaschi A, Luna JM, David A, Uchil PD, MargottinGoguet F, Mothes W, Hazan U, Transy C, Pancino G, Nisole S: Human TRIM gene expression in response to interferons. PLoS One 2009, 4:e4894.

8. Sheehy AM, Gaddis NC, Choi JD, Malim MH: Isolation of a human gene that inhibits HIV-1 infection and is suppressed by the viral Vif protein. Nature 2002, 418:646-650.
9. Neil SJ, Zang T, Bieniasz PD: Tetherin inhibits retrovirus release and is antagonized by HIV-1 Vpu. Nature 2008, 451:425-430.

10. Stremlau M, Owens CM, Perron MJ, Kiessling M, Autissier P, Sodroski J: The cytoplasmic body component TRIM5alpha restricts HIV-1 infection in old world monkeys. Nature 2004, 427:848-853.

11. Laguette N, Sobhian B, Casartelli N, Ringeard M, Chable-Bessia C, Segeral E, Yatim A, Emiliani S, Schwartz O, Benkirane M: SAMHD1 is the dendriticand myeloid-cell-specific HIV-1 restriction factor counteracted by Vpx. Nature 2011, 474:654-657.

12. Hrecka K, Hao C, Gierszewska M, Swanson SK, Kesik-Brodacka M, Srivastava S, Florens L, Washburn MP, Skowronski J: Vpx relieves inhibition of HIV-1 infection of macrophages mediated by the SAMHD1 protein. Nature 2011, 474:658-661.

13. Wilson SJ, Schoggins JW, Zang T, Kutluay SB, Jouvenet N, Alim MA, Bitzegeio J, Rice CM, Bieniasz PD: Inhibition of HIV-1 particle assembly by 2',3'-cyclic-nucleotide 3'-phosphodiesterase. Cell Host Microbe 2012, 12:585-597.

14. Schoggins JW, Wilson SJ, Panis M, Murphy MY, Jones CT, Bieniasz P, Rice CM: A diverse range of gene products are effectors of the type I interferon antiviral response. Nature 2011, 472:481-485.

15. Löchelt M, Romen F, Bastone P, Muckenfuss H, Kirchner N, Kim YB, Truyen U, Rösler U, Battenberg M, Saib A, et al: The antiretroviral activity of APOBEC3 is inhibited by the foamy virus accessory Bet protein. Proc Natl Acad Sci USA 2005, 102:7982-7987.

16. Sawyer SL, Emerman M, Malik HS: Ancient adaptive evolution of the primate antiviral DNA-editing enzyme APOBEC3G. PLOS Biol 2004, 2:E275.

17. Harris RS, Liddament MT: Retroviral restriction by APOBEC proteins. Nat Rev Immunol 2004, 4:868-877.

18. Vartanian JP, Guetard D, Henry M, Wain-Hobson S: Evidence for editing of human papillomavirus DNA by APOBEC3 in benign and precancerous lesions. Science 2008, 320:230-233.

19. Zhang H, Yang B, Pomerantz RJ, Zhang C, Arunachalam SC, Gao L: The cytidine deaminase CEM15 induces hypermutation in newly synthesized HIV-1 DNA. Nature 2003, 424:94-98.

20. Suspene R, Guetard D, Henry M, Sommer P, Wain-Hobson S, Vartanian JP: Extensive editing of both hepatitis B virus DNA strands by APOBEC3 cytidine deaminases in vitro and in vivo. Proc Natl Acad Sci USA 2005, 102:8321-8326.

21. Muckenfuss H, Hamdorf M, Held U, Perkovic M, Löwer J, Cichutek K, Flory E, Schumann GG, Münk C: APOBEC3 proteins inhibit human LINE-1 retrotransposition. J Biol Chem 2006, 281:22161-22172.

22. Suspene R, Aynaud MM, Guetard D, Henry M, Eckhoff G, Marchio A, Pineau P, Dejean A, Vartanian JP, Wain-Hobson S: Somatic hypermutation of human mitochondrial and nuclear DNA by APOBEC3 cytidine deaminases, a pathway for DNA catabolism. Proc Natl Acad Sci USA 2011, 108:4858-4863.

23. Vartanian JP, Sommer P, Wain-Hobson S: Death and the retrovirus. Trends Mol Med 2003, 9:409-413.

24. Moebes A, Enssle J, Bieniasz PD, Heinkelein M, Lindemann D, Bock M, McClure MO, Rethwilm A: Human foamy virus reverse transcription that occurs late in the viral replication cycle. J Virol 1997, 71:7305-7311.

25. Luo K, Wang T, Liu B, Tian C, Xiao Z, Kappes J, Yu XF: Cytidine deaminases APOBEC3G and APOBEC3F interact with human immunodeficiency virus type 1 integrase and inhibit proviral DNA formation. J Virol 2007, 81:7238-7248

26. Guo F, Cen S, Niu M, Saadatmand J, Kleiman L: Inhibition of formulaprimed reverse transcription by human APOBEC3G during human immunodeficiency virus type 1 replication. J Virol 2006, 80:11710-11722.

27. Derse D, Hill SA, Princler G, Lloyd P, Heidecker G: Resistance of human T cell leukemia virus type 1 to APOBEC3G restriction is mediated by elements in nucleocapsid. Proc Natl Acad Sci USA 2007, 104:2915-2920.

28. Li XY, Guo F, Zhang L, Kleiman L, Cen S: APOBEC3G inhibits DNA strand transfer during HIV-1 reverse transcription. J Biol Chem 2007, 282:32065-32074.

29. Bishop KN, Verma M, Kim EY, Wolinsky SM, Malim MH: APOBEC3G inhibits elongation of HIV-1 reverse transcripts. PLOS Pathog 2008, 4:e1000231.

30. Newman EN, Holmes RK, Craig HM, Klein KC, Lingappa JR, Malim MH, Sheehy AM: Antiviral function of APOBEC3G can be dissociated from cytidine deaminase activity. Curr Biol 2005, 15:166-170.

31. Münk C, Jensen BE, Zielonka J, Haussinger D, Kamp C: Running loose or getting lost: how HIV-1 counters and capitalizes on APOBEC3-induced mutagenesis through its Vif protein. Viruses 2012, 4:3132-3161. 
32. Holmes RK, Koning FA, Bishop KN, Malim MH: APOBEC3F can inhibit the accumulation of HIV-1 reverse transcription products in the absence of hypermutation. comparisons with APOBEC3G. J Biol Chem 2007, 282:2587-2595.

33. Münk C, Beck T, Zielonka J, Hotz-Wagenblatt A, Chareza S, Battenberg M, Thielebein J, Cichutek K, Bravo IG, O'Brien SJ, et al: Functions, structure, and read-through alternative splicing of feline APOBEC3 genes. Genome Biol 2008, 9:R48.

34. Albin JS, Harris RS: Interactions of host APOBEC3 restriction factors with HIV1 in vivo: implications for therapeutics. Expert Rev Mol Med 2010, 12:e4.

35. LaRue RS, Andresdottir V, Blanchard Y, Conticello SG, Derse D, Emerman M, Greene WC, Jonsson SR, Landau NR, Löchelt M, et al: Guidelines for naming nonprimate APOBEC3 genes and proteins. J Virol 2009, 83:494-497.

36. Münk C, Willemsen A, Bravo IG: An ancient history of gene duplications, fusions and losses in the evolution of APOBEC3 mutators in mammals. BMC Evol Biol 2012, 12:71.

37. Zielonka J, Münk C: Cellular restriction factors of feline immunodeficiency virus. Viruses 2011, 3:1986-2005.

38. Zielonka J, Marino D, Hofmann H, Yuhki N, Lochelt M, Munk C: Vif of feline immunodeficiency virus from domestic cats protects against APOBEC3 restriction factors from many felids. J Virol 2010, 84:7312-7324.

39. Kolokithas A, Rosenke K, Malik F, Hendrick D, Swanson L, Santiago ML, Portis $J$, Hasenkrug KJ, Evans LH: The glycosylated Gag protein of a murine leukemia virus inhibits the antiretroviral function of APOBEC3. J Virol 2010, 84:10933-10936.

40. Yu X, Yu Y, Liu B, Luo K, Kong W, Mao P, Yu XF: Induction of APOBEC3G ubiquitination and degradation by an HIV-1 Vif-Cul5-SCF complex. Science 2003, 302:1056-1060.

41. Mehle A, Goncalves J, Santa-Marta M, McPike M, Gabuzda D: Phosphorylation of a novel SOCS-box regulates assembly of the HIV-1 Vif-Cul5 complex that promotes APOBEC3G degradation. Genes Dev 2004, 18:2861-2866.

42. Bogerd HP, Doehle BP, Wiegand HL, Cullen BR: A single amino acid difference in the host $A P O B E C 3 G$ protein controls the primate species specificity of HIV type 1 virion infectivity factor. Proc Natl Acad Sci USA 2004, 101:3770-3774.

43. Katzourakis A, Gifford RJ, Tristem M, Gilbert MT, Pybus OG: Macroevolution of complex retroviruses. Science 2009, 325:1512.

44. Russell RA, Wiegand HL, Moore MD, Schafer A, McClure MO, Cullen BR: Foamy virus Bet proteins function as novel inhibitors of the APOBEC 3 family of innate antiretroviral defense factors. J Virol 2005, 79:8724-8731.

45. Perkovic M, Schmidt S, Marino D, Russell RA, Stauch B, Hofmann H, Kopietz F, Kloke BP, Zielonka J, Strover H, et al: Species-specific inhibition of APOBEC3C by the prototype foamy virus protein bet. J Biol Chem 2009, 284:5819-5826.

46. Cullen BR: Role and mechanism of action of the APOBEC3 family of antiretroviral resistance factors. J Virol 2006, 80:1067-1076.

47. Chareza S, Slavkovic Lukic D, Liu Y, Räthe AM, Münk C, Zabogli E, Pistello M, Löchelt M: Molecular and functional interactions of cat APOBEC3 and feline foamy and immunodeficiency virus proteins: different ways to counteract host-encoded restriction. Virology 2012, 424:138-146.

48. Alke A, Schwantes A, Zemba M, Flügel RM, Löchelt M: Characterization of the humoral immune response and virus replication in cats experimentally infected with feline foamy virus. Virology 2000, 275:170-176.

49. Löchelt M: Foamy virus transactivation and gene expression. Curr Top Microbiol Immunol 2003, 277:27-61.

50. Han GZ, Worobey M: An endogenous foamy-like viral element in the coelacanth genome. PLoS Pathog 2012, 8:e1002790.

51. Alke A, Schwantes A, Kido K, Flötenmeyer M, Flügel RM, Löchelt M: The bet gene of feline foamy virus is required for virus replication. Virology 2001, 287:310-320.

52. Rethwilm A: The replication strategy of foamy viruses. Curr Top Microbiol Immunol 2003, 277:1-26.

53. Lindemann D, Rethwilm A: Foamy virus biology and its application for vector development. Viruses 2011, 3:561-585.

54. Rethwilm A: Molecular biology of foamy viruses. Med Microbiol Immunol 2010, 199:197-207

55. Heneine W, Schweizer M, Sandstrom P, Folks T: Human infection with foamy viruses. Curr Top Microbiol Immunol 2003, 277:181-196.

56. Switzer WM, Salemi M, Shanmugam V, Gao F, Cong ME, Kuiken C, Bhullar V, Beer BE, Vallet D, Gautier-Hion A, et al: Ancient co-speciation of simian foamy viruses and primates. Nature 2005, 434:376-380.
57. Materniak M, Hechler T, Löchelt M, Kuzmak J: Similar patterns of infection with bovine foamy virus in experimentally inoculated calves and sheep. J Virol 2013, 87:3516-3525.

58. Bailey TL, Williams N, Misleh C, Li WW: MEME: discovering and analyzing DNA and protein sequence motifs. Nucleic Acids Res 2006, 34:W369-W373.

59. Kobayashi M, Takaori-Kondo A, Miyauchi Y, Iwai K, Uchiyama T: Ubiquitination of APOBEC3G by an HIV-1 Vif-Cullin5-Elongin B-Elongin C complex is essential for Vif function. J Biol Chem 2005, 280:18573-18578.

60. Mehle A, Strack B, Ancuta P, Zhang C, McPike M, Gabuzda D: Vif overcomes the innate antiviral activity of APOBEC3G by promoting its degradation in the ubiquitin-proteasome pathway. J Biol Chem 2004, 279:7792-7798.

61. Conticello SG, Harris RS, Neuberger MS: The Vif protein of HIV triggers degradation of the human antiretroviral DNA deaminase APOBEC3G. Curr Biol 2003, 13:2009-2013.

62. Marin M, Rose KM, Kozak SL, Kabat D: HIV-1 Vif protein binds the editing enzyme APOBEC3G and induces its degradation. Nat Med 2003, 9:1398-1403.

63. Chivian D, Kim DE, Malmstrom L, Bradley P, Robertson T, Murphy P, Strauss CE, Bonneau R, Rohl CA, Baker D: Automated prediction of CASP-5 structures using the Robetta server. Proteins 2003, 53(Suppl 6):524-533.

64. Kim DE, Chivian D, Baker D: Protein structure prediction and analysis using the Robetta server. Nucleic Acids Res 2004, 32:W526-W531.

65. Benkert $P$, Kunzli M, Schwede T: QMEAN server for protein model quality estimation. Nucleic Acids Res 2009, 37:W510-W514.

66. Wallner B, Elofsson A: Can correct protein models be identified? Protein Sci 2003, 12:1073-1086.

67. Romen F, Pawlita M, Sehr P, Bachmann S, Schröder J, Lutz H, Löchelt M: Antibodies against Gag are diagnostic markers for feline foamy virus infections while Env and Bet reactivity is undetectable in a substantial fraction of infected cats. Virology 2006, 345:502-508.

68. Weikel J, Löchelt $M$, Truyen U: Demonstration of feline foamy virus in experimentally infected cats by immunohistochemistry. J Vet Med A Physiol Pathol Clin Med 2003, 50:415-417.

69. Zemba M, Alke A, Bodem J, Winkler IG, Flower RL, Pfrepper K, Delius H, Flügel RM, Löchelt M: Construction of infectious feline foamy virus genomes: cat antisera do not cross-neutralize feline foamy virus chimera with serotype-specific Env sequences. Virology 2000, 266:150-156.

70. Yon J, Fried M: Precise gene fusion by PCR. Nucleic Acids Res 1989, 17:4895.

71. Rasband WS: ImageJ. U.S. Bethesda, Maryland, USA: National Institutes of Health; 2011. http://imagej.nih.gov/ij/, 1997.

\section{doi:10.1186/1742-4690-10-76}

Cite this article as: Slavkovic Lukic et al:: Identification of the feline foamy virus Bet domain essential for APOBEC3 counteraction.

Retrovirology 2013 10:76.

\section{Submit your next manuscript to BioMed Central and take full advantage of:}

- Convenient online submission

- Thorough peer review

- No space constraints or color figure charges

- Immediate publication on acceptance

- Inclusion in PubMed, CAS, Scopus and Google Scholar

- Research which is freely available for redistribution

Submit your manuscript at www.biomedcentral.com/submit 